\title{
Cue usage in memory for location when orientation is fixed
}

\author{
Sylvia Fitting, Douglas H. Wedell, and Gary L. Allen \\ University of South Carolina, Columbia, South Carolina
}

\begin{abstract}
In previous research, it was demonstrated that including one or three cues surrounding a circular field had no effect on spatial memory for dot locations when the field's orientation was fixed, but that there were very large effects when orientation varied across trials (Fitting, Wedell, \& Allen, 2007). In four new experiments, we explored the use of external cues in the fixed orientation environment, using $0,4,8$, or 24 cues and manipulating task difficulty. In Experiments 1-3, the angular bias data supported the use of four quadrant-based prototypes regardless of cue condition, but there were clear cue effects on radial prototype locations. Increasing the number of cues enhanced accuracy of spatial memory for targets closer to cues. In Experiment 4, we severely challenged memory by using multiple targets and a filled delay before estimation. Doing so demonstrated an effect of cues on the categorical structuring of memory. Collectively, findings indicate that when orientation is fixed, cues bolster fine-grain memory, but that they only alter the default categorical scheme when memory demands are high.
\end{abstract}

Place-based memory is a multifaceted phenomenon that can involve a number of different processes driven by task demands and available information-even in tasks involving nothing more than specifying a location seen seconds earlier. One well-established distinction is that memory for location may be coded at two different levels: a finegrain and a categorical representation (Allen \& Haun, 2004; Easton \& Sholl, 1995; Huttenlocher, Hedges, \& Duncan, 1991; Mou \& McNamara, 2002; Wang \& Spelke, 2000). Fine-grain information may be conceived as metrically veridical, based on a coordinate frame of reference (for example, polar coordinates), and it is postulated to be very fragile in nature and short-lived (Haun, Allen, \& Wedell, 2005; Hund \& Plumert, 2002; Spencer \& Hund, 2002). In contrast, categorical information is more robust and based on properties of the task field. As uncertainty of fine-grain memory increases, estimates of remembered locations are drawn more strongly toward the center of spatial categories, resulting in an unmistakable pattern of bias (Huttenlocher et al., 1991).

How might these coding processes be influenced by the presence of external cues in a task in which a stationary observer remembers the location of a position within a geometric field? To date, little research has examined this question. However, such situations occur fairly often, such as when observers look for faces in a crowd, for blips on a radar screen, or even for objects in the sky. The issue of how cues in the visual environment may determine the categorical structure or influence the fine-grain memory representation requires clarification and is the focus of our investigation.
One experimental paradigm that has been useful in studying fine-grain and categorical memory processes is the dot location task (Huttenlocher et al., 1991), in which a dot is presented in a circular field of fixed orientation; then, after a brief delay, the observer is asked to reproduce the location. When cues are absent, the general pattern of results observed in this paradigm is that estimates are biased toward the center of the quadrant in which the dot was presented. The results are consistent with the idea that observers use a polar coordinate system and impose horizontal and vertical axes on the circle to define boundaries. The resulting quadrants act, then, as categories, and the interior point within each quadrant serves as a category prototype. Although this default category structure induces systematic bias in estimation, it reduces overall error in estimation, as demonstrated by Huttenlocher et al.

Recently, we have examined the role of cues in memory for location by using this task and varying the number of available external reference cues (Fitting, 2005; Fitting, Wedell, \& Allen, 2007). Participants were asked to reproduce locations of presented dots in a circular task field, with 0,1 , or 3 external reference cues available. The basic finding was that when orientation to the task field was fixed on all trials, there was no evidence for a cue-based categorization scheme. However, when orientation varied from trial to trial, categorical encoding was centered on the external cues, reflecting the use of a cue-based representation. These results parallel to some degree those found for the Morris water maze (Morris, 1981). When orientation is fixed by using a constant starting point, the rat appears to locate the hidden platform via response learning and,

S. Fitting, sfitting@vcu.edu 
hence, can ignore cues. However, under variable orientation (i.e., variable starting places), the animal must rely on an allocentric representation that is dependent on coding the platform's location relative to external cues.

The lack of cue effects found by Fitting et al. (2007) in the fixed orientation version of the dot location task provides evidence for a cue-independent spatial encoding process. However, it is not clear whether participants ignored the peripheral cues as a default for fixed orientation tasks or as an adaptive behavior, given the specific cue structures used in that experiment. The number of cues used in that experiment supported fewer categories than the default four-category structure. Because using fewer categories theoretically should result in greater bias and greater absolute error, the failure to use the external cues in that experiment may simply reflect adaptive behavior.

The present studies examined the potential use of cues in a fixed environment when it should be adaptive to do so. First, we examined whether cues could have the potential to redefine the structure of an environmental task field, as reflected in changes in the pattern of angular bias. Our previous work that manipulated the number of cues supported the maintenance of the default structure when the task field was static (Fitting et al., 2007). Further support for the maintenance of the default structure is given in another study that varied the shape of the response field (square, pentagon, and triangle), with the finding that the same four basic quadrants for encoding location were used (Wedell, Fitting, \& Allen, 2007). However, the manipulations in both studies may not have afforded a category structure that could adaptively reduce error. In contrast, the present experiments did so by including many more cues (i.e., 4, 8, and 24) and by examining the effects of cue manipulation under memory-taxing conditions. These conditions provide a strong test of the adaptive use of cues to restructure memory for location.

Second, we examined whether cues can be used to anchor fine-grain memory, reducing error as indexed by measures of absolute error and inconsistency of estimates. Recent studies have demonstrated a much lower error variance for targets located near landmark cues (Werner \& Diedrichsen, 2002). Similarly, Fitting, Wedell, and Allen (in press) found reduced error for targets located near a cue when the task field was rotated. On the basis of these results, we hypothesized that even if additional cues are not used to establish spatial categories, they may be used as anchors to stabilize fine-grain memory and to reduce absolute error in estimation. These effects should be dependent on the time allocated for encoding and the delay before retrieval. In particular, these effects may be strongest at longer delays, since findings of previous studies suggest that memory for locations becomes less certain as the delay between learning and reproducing the locations increases (Hund \& Plumert, 2002; Huttenlocher et al., 1991; Spencer \& Hund, 2003). The manipulations of the number of cues and timing in the present set of experiments tested for the adaptive use of cues in reducing memory error that might be attributable to cues bolstering fine-grain memory.

Overall, the conceptual rationale for this investigation was to understand better how cues are used when orien- tation to the environment is fixed. A shift in encoding strategy to incorporate cues is consistent with the idea of flexibility in spatial cognition (Simmering, Spencer, \& Schöner, 2006; Spencer, Simmering, \& Schutte, 2006). Hund and Plumert (2005) demonstrated how stability and flexibility in forming category structures varies developmentally. Our interest in exploring the notion of the flexibility of spatial memory centers on whether participants switch the default encoding scheme of quadrant-based encoding within a fixed environment. In addition, we explore the general framework of the rational or adaptive basis of memory (Huttenlocher et al., 1991), assuming that failure to engage a cue-based framework would generally lead to much greater errors. In this vein, one possibility is that cues may be used to anchor fine-grain memory and to reduce error, but not to alter the representation used in categorical memory. Before describing the present set of experiments, we will briefly review details of prior research and modeling approaches.

\section{Fuzzy-Boundary Model for Inferring Spatial Categories}

In order to determine whether cues are affecting the category structure, one needs a model for inferring the category structure from the pattern of responses. The model we use is based on our earlier work (Fitting, Wedell, \& Allen, 2005, 2007), in which we extended Huttenlocher et al.'s (1991) category-adjustment model in two basic ways. First, the model included parameters that reflect the tendency to recruit prototypes from adjacent categories in making estimates of targets located near a boundary. Because targets can recruit prototypes across boundaries, we use the term "fuzzy boundaries" to refer to this version of the model. This model is similar to the uncertain boundaries version of the category-adjustment model developed by Huttenlocher et al., except that it does not require a priori determination of boundaries but can infer these from prototype locations. Second, the model included two possible bases for categorization: one in which categories are based on a cue-independent segmentation of the task field, and one in which categories are centered on available peripheral cues. The former model is referred to as the fixed-quadrants fuzzy-boundary model and the latter as the flexible fuzzy-boundary model. A detailed description of the development of these two fuzzy-boundary models is presented elsewhere (Fitting et al., 2005, 2007). Here, we briefly review relevant aspects of the model development. The Appendix provides a short description of the equations underlying these two models.

These models are based on the basic category-adjustment model that was described by Huttenlocher et al. (1991), with memory for spatial location being represented at two levels of detail: a fine-grain value and a categorical value. According to the category-adjustment model, the expected value of the response in an estimation task $(E[R])$ is characterized as a weighted average of fine-grain and categorical information. The expected bias ( $E[\mathrm{Bias}])$ is then determined by subtracting the actual value from the response and, thus, the expected bias is characterized by the following equation: 


$$
E[\text { Bias }]=E[R]-\mu=\lambda \mu+(1-\lambda) p-\mu,
$$

where $\mu$ is the mean of the distribution of fine-grain memory values for the object assumed to be unbiased and hence equated with the true location of the object. Similarly, $p$ is the mean of the distribution of prototype locations for the relevant category. The parameter, $\lambda$, which varies from 0 to 1 , represents the relative weight of the fine-grain information. This model provides a good approximation to the angular and radial biases typically observed in the dot location task (i.e., four quadrant-based categories defined by borders running along horizontal and vertical axes), accounting for the shifts in estimation toward the prototype locations. Although Spencer and Hund (2002) developed an alternative explanation of this bias pattern based on shifts away from category boundaries rather than toward category prototypes, our theorizing and modeling within this article will follow the prototype-based representation of the process.

Applying the polar coordinate representation typically employed for this task, the models predict patterns of angular bias as a function of category structure. The differences in the predictions of these models are illustrated in Figure 1. Predictions of angular bias from the fixed-quadrants fuzzy-boundary model are shown in panel A of Figure 1. The two different curves reflect different degrees of fuzziness, as determined by the $c$ parameter of the model (see the Appendix). The key idea here is that regardless of number of cues, the bias pattern is one in which the negatively sloping functions intersect the origin once in each quadrant, indicating the location of the category prototypes. Panels B and C indicate the flexible fuzzy-boundary model predictions based on 1 cue at $305^{\circ}$ (panel B) or 3 cues at $80^{\circ}, 170^{\circ}$, and $305^{\circ}$ (panel C). The model in this case places the prototypes at the locations of the cues. The pattern of bias is strikingly different for the fixed-quadrant fuzzy-boundary version of the model (panel A) and the flexible version of the model based on different number of cues (panels B and C). The panels of Figure 1 correspond roughly with the results of Fitting et al. (2007). Data in the fixed orientation are best described by panel A (default structure), and data in the dynamic task environment are best described by panels $\mathrm{B}$ and $\mathrm{C}$ (cue-based structure).

\section{Overview of Experiments}

Experiment 1 used the cue structure illustrated in panel B of Figure 2. Note that the 4 cues provide the basis for four categories, so that the increased error associated with using fewer categories is no longer a disadvantage. As is illustrated in panels A and B of Figure 2, the categorization structure based on the four cues differs strongly from that implied by the default structure, since the categories are rotated $45^{\circ}$. This manipulation then maximizes the effect size for angular bias and provides a powerful test of the effects of including external cues on participants' estimations of dot location from memory.

In Experiment 2, we increased the number of available reference cues surrounding the circular task field to 8 , as is illustrated in panel $\mathrm{C}$ of Figure 2. Experiment 2 provides a test of the impact of providing enough available cues

\section{A Fixed-Quadrants Fuzzy-Boundary Model}

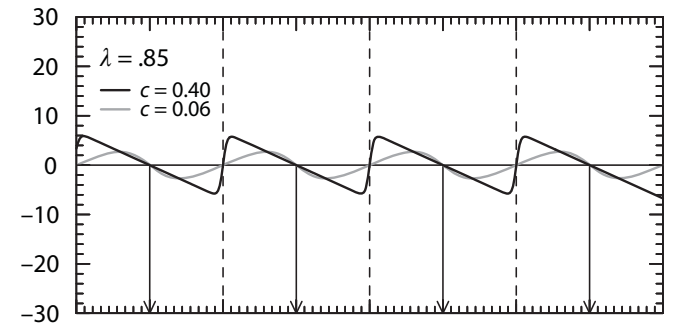

\section{B Cue-Based Fuzzy-Boundary Model (1 Cue)}

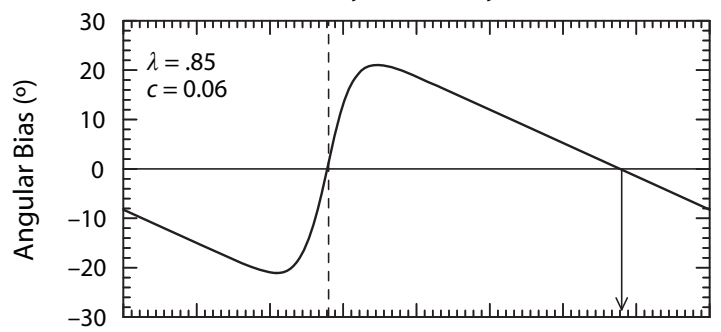

\section{Cue-Based Fuzzy-Boundary Model (3 Cues)}

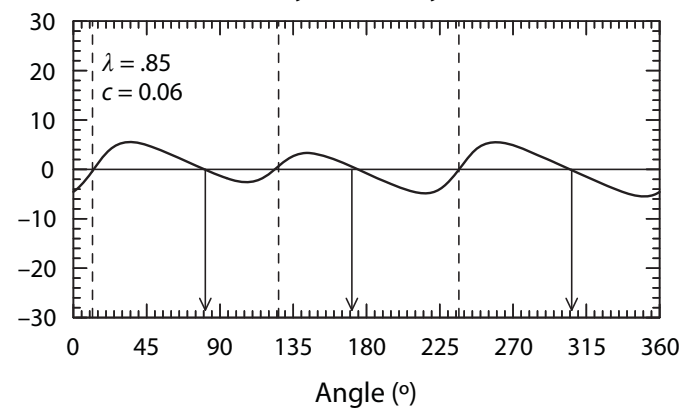

Figure 1. (A) The fixed-quadrants fuzzy-boundary model with the same fixed boundaries and quadrant prototypes as those suggested by the category-adjustment model. Note that when the sensitivity parameter $(c)$ gets larger, it is nearly identical to the model by Huttenlocher et al. (1991). (B, C) Predictions of the flexible fuzzy-boundary model, with prototypes equated with cue location, probabilistic recruitment of prototypes across categories, and inferred boundaries located halfway between adjacent prototypes. Panel B: One cue case with the prototype at $305^{\circ}$ and the boundary at $125^{\circ}$. Panel C: Three prototypes located at $80^{\circ}$, $170^{\circ}$, and $305^{\circ}$, with inferred boundaries at $237.5^{\circ}, 12.5^{\circ}$, and $125^{\circ}$. $\lambda$, weighting of fine-grain information. The arrows in all three panels indicate the predicted prototype locations by the model.

to support a more detailed categorization scheme. Here, our flexible fuzzy-boundary model predicts that if participants were able to use the full set of cues to determine spatial categories, then the pattern of bias would reflect eight prototypes, and the degree of bias and absolute error would be greatly reduced.

In Experiment 3, either 0 or 24 reference cues were presented, with the presentation time of the target and the masked delay time being manipulated between subjects. The manipulation of presentation and delay time was included to show whether these two factors play a role in cue 


\section{Cues}

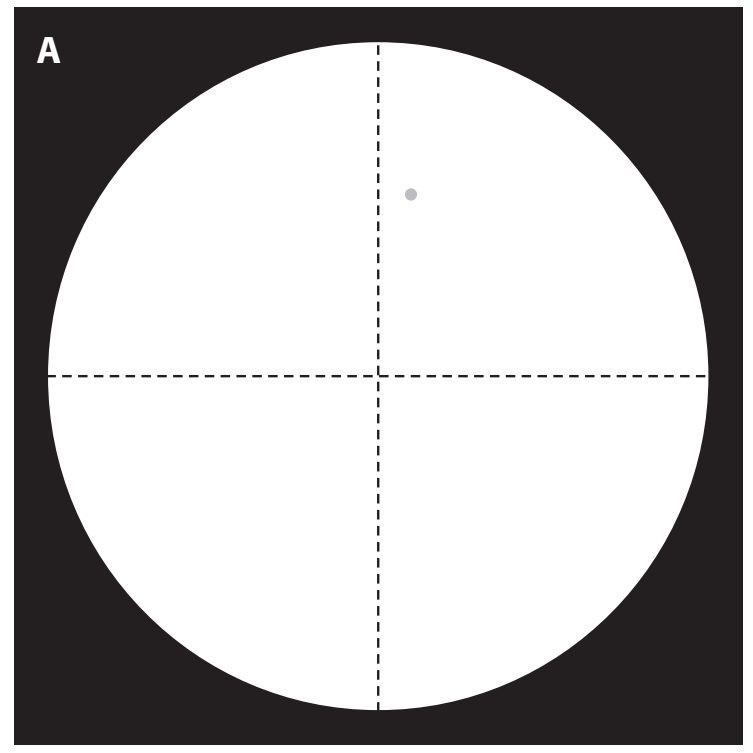

\section{Cues}

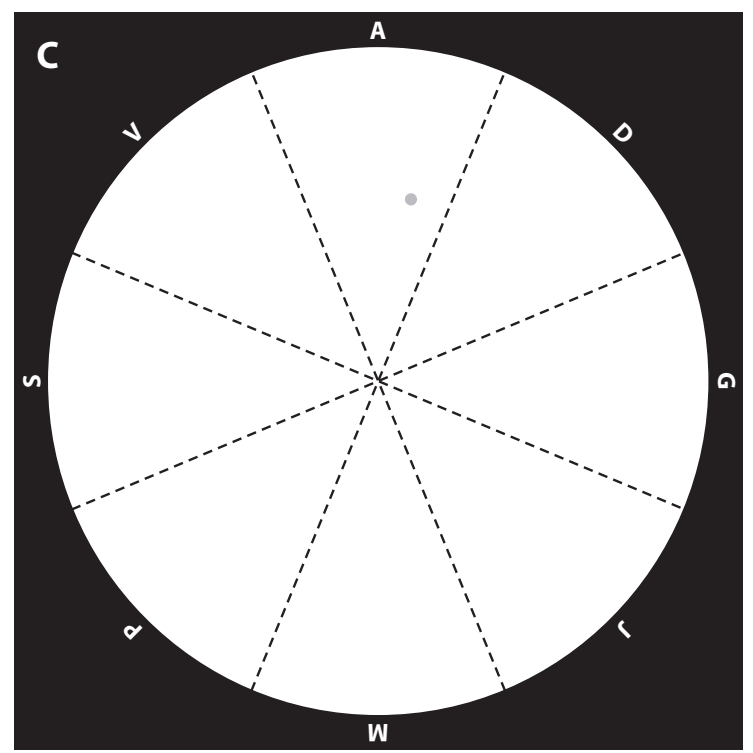

\section{Cues}

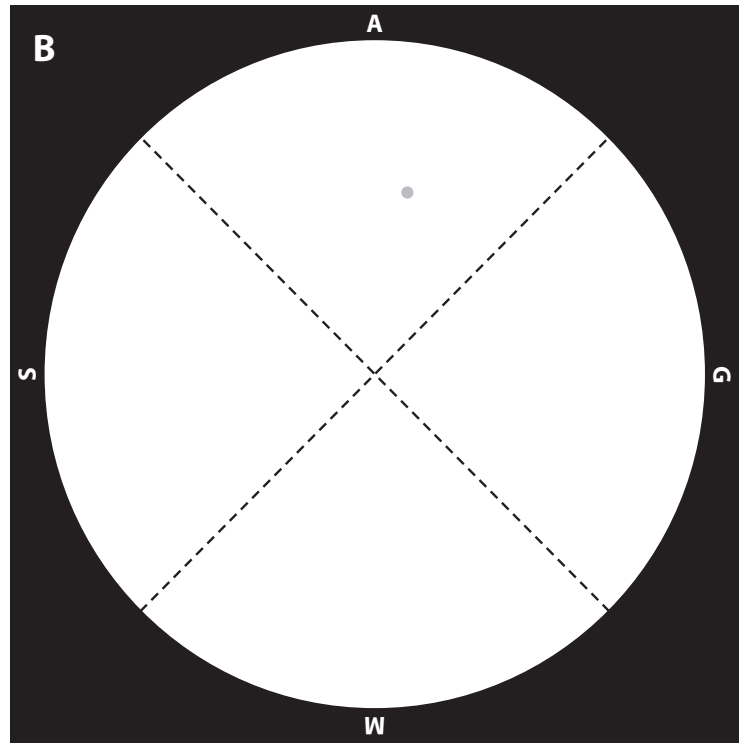

\section{Cues}

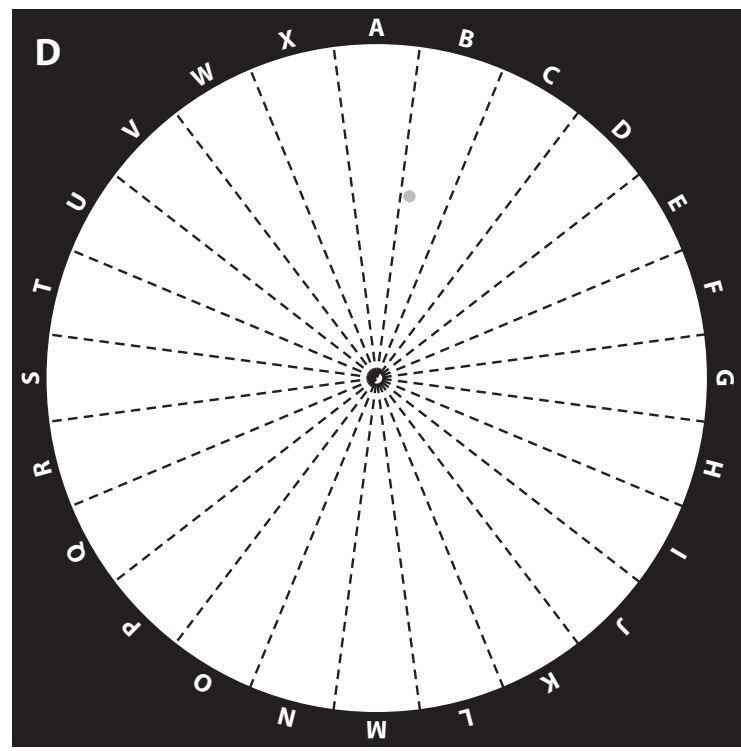

Figure 2. (A) The viewer-based frame of reference as predicted by the fixed-quadrants fuzzy-boundary model, with the imposed horizontal and vertical line as fixed boundaries imposed on the circular field. (B, C, D) The cue-based frame of reference as predicted by the flexible fuzzy-boundary model with the formed categories depending on the number of available external reference cues, indexed by the letters surrounding the circular task field. The dashed lines were not visually presented on the display to the participants.

effects on categorical memory and fine-grain memory. We hypothesized that an increase in presentation time of the dot location provides a more stable representation, leading to increased accuracy of memory for the object location. We also hypothesized that increasing the delay time would result in greater reliance on categorical memory and, hence, greater bias effects, as was found in previous work (Hund \& Plumert, 2002; Merchant, Fortes, \& Georgopoulos, 2004; Spencer \& Hund, 2002).

Experiment 4 used the same cue structure as that in Experiment 1 ( 0 and 4 cues), but greatly increased memory demands. First, four color-coded target locations were presented sequentially on each trial. Second, before participants were asked to estimate one of these four dot loca- 
tions, they were asked to solve a Shepard-Metzler mental rotation task for at least $10 \mathrm{sec}$, engaging spatial working memory resources and thereby making it difficult to hold the location in memory. Thus, in Experiment 4, we tested whether increased working memory load and memory demands may shift the categorization structure to be based on cues rather than on quadrants.

\section{EXPERIMENT 1}

There are two ways in which Experiment 1 provides a stronger test of the potential use of external cues than did our previous research (Fitting et al., 2007). First, in the earlier study, the number of cues was manipulated between subjects rather than within subjects. The present use of a within-subjects manipulation of this factor provides greater power to test for the effects of cues. Second, the previous manipulation provided either 1 or 3 external cues. If these cues were used to determine category prototypes, then the direction of angular bias would change drastically, but so would the amount of bias. Thus, given competing category representations, participants may have simply chosen the one that would yield less bias. In Experiment 1, we used 4 cues so that the competing representation would yield a comparable amount of bias, since both representations would be based on four evenly spaced categories. The location of the cues - as shown in panel B of Figure 2 - provides a maximum shift of the prototype locations and, hence, a good test of the representation being used. If participants use cues as prototypes, then angular bias should be very poorly fit by the fixed-quadrants fuzzy-boundary model and should require the flexible fuzzy-boundary model with prototypes being placed at the cue locations.

\section{Method}

Participants and Design. Twenty undergraduate students from the University of South Carolina psychology department participant pool participated in the experiment in exchange for course credits. The same participant pool was used in all four experiments. The design consisted of three within-subjects factors: cue ( 0 vs. 4$)$, radius (short vs. long), and angle $\left(3^{\circ}, 25^{\circ}, 43^{\circ}\right.$, and $75^{\circ}$ in each of the four quadrants). Trials were presented in a randomized sequence.

Task. Parameters of the task were the same as those in previous research (Fitting et al., 2007). All participants attempted to reproduce the locations of 32 dots located within a circular region centered on a computer display. The 32 targets were presented twice and distributed over the circular area in order to provide sufficient data for modeling the predicted bias and to gather a measure of the unreliability of repeated estimates. Sixteen dots were located at a radius of 92 pixels (short radius from the center), and the other 16 dots were located at a radius of 168 pixels (long radius). Each target appeared at one of four different angles $\left(3^{\circ}, 25^{\circ}, 43^{\circ}\right.$, and $\left.75^{\circ}\right)$ within one of the four quadrants. Targets were presented successively in random order. In contrast with our previous experiments, the peripheral reference cues used in the present study were white letters, which gave them distinctiveness and enabled them to be quickly encoded. The two cue conditions consisted of 0 or 4 reference cues that were $90^{\circ}$ away from each other, as shown in Figure 2 (panels A and B).

Four dependent variables were used to examine spatial memory. Angular bias was measured in degrees by subtracting the angle of the actual location from the angle of the reproduced location. A negative value indicates a clockwise bias, and a positive value indicates a counterclockwise angular bias. Radial bias was measured in pixels by subtracting the radial distance of the actual point from the radial distance of the observed point. A negative value indicates a radial bias toward the center of the circle, and a positive value indicates a radial bias away from the center. We used two other measures of error. Absolute error - the Euclidean distance between actual to estimated dot locations - was used as a global measure of unsigned error or inaccuracy of place memory. However, because absolute error has the disadvantage of being driven by both systematic error (i.e., bias) and unsystematic error, we developed an alternative error measure based on the inconsistency of estimation, which is the Euclidean distance between the participant's two estimates of a location within a given condition. Inconsistency of estimation, which is similar to the measure of variable error (Spencer \& Hund, 2003), has the advantage of being independent of the two bias measures and, hence, is more clearly linked to inaccuracy of fine-grain memory representation. We had not used it in previous studies, because it requires at least two estimates of the same dot location within the same condition.

Materials and Apparatus. All materials and instructions were presented via microprocessors with 15 -in. (38-cm) monitors. The white circular region was presented on a black background in video graphics array mode at a resolution of $640 \times 480$ pixels, with a radius of 212 pixels, as shown in Figure 2 (but without the dashed lines). A red dot that was 5 pixels in diameter was presented within the circular task field on each trial. No reference cues surrounded the circular task field in the 0 -cue condition, whereas 4 external reference cues were located along the circular region in the 4-cue condition. The external reference cues were the letters $\mathrm{A}, \mathrm{G}, \mathrm{M}$, and $\mathrm{S}$, which were located in a clockwise order every $90^{\circ}$ starting from the top of the circular field (see Figure 2). The letters A, G, M, and S were used because they represent the same cue locations as those in Experiments 2 and 3.

Procedure. Groups of 1-5 participants were tested at the same time in a laboratory room with computer terminals spaced approximately $1 \mathrm{~m}$ apart. After reading the general instructions, participants experienced an initial learning set of 2 trials with feedback, and a second learning set of 5 trials without feedback. This was followed by the actual test that included a total of 128 trials with 32 different dot locations that were presented twice in each cue condition. No feedback was given for any of the test trials. Each dot was on-screen for $1 \mathrm{sec}$, then it was covered by a dynamic checkerboard mask for $4.5 \mathrm{sec}$, followed by a blank circle. The checkerboard mask consisted of white and black $10 \times$ 10 pixel squares that were exchanged every $0.25 \mathrm{sec}$, creating a moving pattern to avoid fixation. A crosshairs cursor appeared at the center of the circle, serving as a marker for the participant to indicate the to-beremembered dot location on the response display by using the mouse. Responses were recorded in pixel units of the marked locations.

\section{Results}

Data analyses. The observed dot locations were used to generate the dependent variables in all analyses. Because outliers can obscure systematic effects, we eliminated data points that were more than 2 standard deviations from the mean on absolute error and angular bias separately for each radius and cue condition. These were replaced by the mean of the remaining values for the specific dot location within each condition. In the 0 -cue condition, $6.25 \%$ of the data were replaced for both the short and the long radius. In the 4-cue condition, $7.50 \%$ were replaced for the short radius, and $4.06 \%$ for the long radius. An $\alpha=.05$ was used to determine the significance for all statistical tests. Violations of compound symmetry were addressed via the use of the Greenhouse-Geisser degrees of freedom correction factor (Greenhouse \& Geisser, 1959). For each dependent variable, a 2 (cue condition: 0 vs. 4 ) $\times 2$ (radius: short vs. long) $\times 16$ (angles: $3^{\circ}, 25^{\circ}, 43^{\circ}$, and $75^{\circ}$ in each of the four quadrants) within-subjects factorial ANOVA was conducted, and the results are displayed in Table 1 (for Experiment 1). 
Table 1

Degrees of Freedom $(d f)$ and $F$ Values for 2 (Cue Condition) $\times$ 2 (Radius) $\times 16$ (Actual Angle) Within-Subjects Factorial ANOVAs

\begin{tabular}{|c|c|c|c|c|c|c|}
\hline Source & $d f$ & $\begin{array}{l}\text { Angular } \\
\text { Bias }\end{array}$ & $\begin{array}{l}\text { Residual on } \\
\text { FQFBM }\end{array}$ & $\begin{array}{l}\text { Radial } \\
\text { Bias }\end{array}$ & $\begin{array}{l}\text { Absolute } \\
\text { Error }\end{array}$ & $\begin{array}{l}\text { Inconsistency } \\
\text { of Estimation }\end{array}$ \\
\hline \multicolumn{7}{|c|}{ Experiment 1} \\
\hline Cue (C) & $(1,19)$ & 0.06 & 0.06 & 0.04 & $5.18^{*}$ & $6.59^{*}$ \\
\hline Radius (R) & $(1,19)$ & 0.13 & 0.13 & $25.43^{* * *}$ & $7.60^{*}$ & 0.34 \\
\hline Angle (A) & $(15,285)$ & $6.80^{* * *}$ & 0.72 & $4.12^{* *}$ & 1.24 & 0.88 \\
\hline $\mathrm{C} \times \mathrm{R}$ & $(1,19)$ & 0.14 & 0.14 & 0.24 & 0.52 & 0.15 \\
\hline $\mathrm{C} \times \mathrm{A}$ & $(15,285)$ & 0.89 & 0.89 & 1.42 & 0.93 & 1.09 \\
\hline $\mathrm{R} \times \mathrm{A}$ & $(15,285)$ & 1.80 & 1.80 & 1.47 & 0.86 & 0.88 \\
\hline $\mathrm{C} \times \mathrm{R} \times \mathrm{A}$ & $(15,285)$ & 1.41 & 1.41 & 0.63 & 0.81 & 0.93 \\
\hline \multicolumn{7}{|c|}{ Experiment 2} \\
\hline $\mathrm{C}$ & $(1,19)$ & 0.19 & 0.27 & $4.49^{*}$ & $5.30^{*}$ & $10.87^{* *}$ \\
\hline $\mathrm{R}$ & $(1,19)$ & 0.44 & 1.58 & $85.31^{* * * *}$ & $54.60^{* * *}$ & $34.36^{* * *}$ \\
\hline $\mathrm{A}$ & $(15,285)$ & $8.48^{* * *}$ & 1.11 & $5.73^{* * *}$ & $2.30^{*}$ & 0.53 \\
\hline $\mathrm{C} \times \mathrm{R}$ & $(1,19)$ & 0.53 & 2.50 & 0.03 & $8.78^{* *}$ & 3.61 \\
\hline $\mathrm{C} \times \mathrm{A}$ & $(15,285)$ & $2.45^{*}$ & 0.61 & 0.88 & 1.32 & 1.39 \\
\hline $\mathrm{R} \times \mathrm{A}$ & $(15,285)$ & $2.54^{* *}$ & 1.80 & 1.33 & 1.61 & 0.85 \\
\hline $\mathrm{C} \times \mathrm{R} \times \mathrm{A}$ & $(15,285)$ & 1.44 & 1.36 & 1.30 & 0.49 & 1.40 \\
\hline
\end{tabular}

Note-FQFBM, fixed-quadrants fuzzy-boundary model. Residuals based on a 6-parameter model in Experiment 1 and on an 11-parameter model in Experiment 2. ${ }^{*} p<.05 .{ }^{* *} p<$ $.01{ }^{* * *} p<.001$

Angular bias. If cues were used as category centroids or prototypes, then the angular bias measure should have shown a strong interaction of cue with angle. For angular bias, no significant cue effect or interaction with cue was noted, indicating that cue did not influence angular bias (see Table 1 for Experiment 1). As was expected, a significant angle effect was observed. To analyze angular bias more carefully, we conducted a trend analysis of angle within each quadrant. The linear trend was significant $[F(1,19)=35.2$, $p<.001]$ and consistent with both the simple categoryadjustment model and the fuzzy-boundary version of the model. The quadratic trend was also significant $[F(1,19)=$ 23.9, $p<.001]$ and consistent with predictions from the fuzzy-boundary version of the model.

Because no cue effect or interaction with cue was noted, the mean angular bias scores were combined for the 0 -cue and 4-cue conditions in fitting the fuzzy-boundary model to the data. Different versions of this model can be generated by freeing different parameter values. Modeling of the two sets of 32 target means was accomplished using the iterative nonlinear regression procedure within SYSTAT (Wilkinson, 1989), with a least-squares error criterion and the Gauss-Newton method of steepest descent. Parameters were eliminated as long as $R^{2}$ did not drop significantly. As is shown in Figure 3A, a six-parameter fixedquadrants fuzzy-boundary model fit the data adequately $\left(R^{2}=.66\right)$. As was expected, prototypes were modeled as being located centrally within each quadrant $\left(p_{1}=\right.$ $\left.53.24^{\circ}, p_{2}=148.34^{\circ}, p_{3}=212.50^{\circ}, p_{4}=313.13^{\circ}\right)$. The value of fine-grain memory weight $(\lambda=.907)$ was fairly high, leading to only modest bias effects. The value of the fuzzy-boundary parameter $(c=0.086)$ indicated fairly sharp boundaries. As a further test of the model's ability to explain the data, a three-way within-subjects factorial ANOVA on the residuals was conducted after subtracting model predictions from each participant's responses. As is shown in Table 1, the significant effect of angle was elimi- nated, indicating that the six-parameter fixed-quadrants fuzzy-boundary model provided a good description of the pattern of bias. Thus, the data were adequately described by the same four quadrant-based prototypes, regardless of cue condition.

Radial bias. No cue effect or interaction with cue was noted, but there were significant main effects for radius and angle (see Table 1 for Experiment 1). The significant main effect of radius indicated a radial bias toward the circumference for the short radius $(M=4.47)$ and in the opposite direction for the long radius $(M=-1.96)$. This pattern reflects a radial prototype located between short and long-radius targets. The only other effect on radial bias was that of angle. Much of this effect appeared to be due to a tendency to show a leftward bias. Mean radial bias was significantly greater for estimates in the left half of the circle $(M=3.19)$ than in the right half $(M=-0.62)$, consistent with a leftward tendency in estimation.

Absolute error and inconsistency of estimation. Absolute error reflects the distance (in pixels) between the estimated and actual locations. As such, it is a measure of inaccuracy of estimation. Table 1 indicates significant main effects for cue and radius. The significant cue effect revealed reduced error for the 4-cue condition $(M=13.37)$ as compared with the 0 -cue condition $(M=$ 14.63). The significant radius effect revealed slightly reduced error for the long radius $(M=13.25)$ as compared with the short radius $(M=14.75)$.

One problem interpreting absolute error is that it includes effects of systematic error, or bias, as well as effects of nonsystematic error. To obtain a measure of error that is independent of bias effects, we calculated the absolute distance between the participant's first estimate and second estimate of a dot location as a measure of the inconsistency of estimation. Because this measure does not reflect bias, we believe it to be a clearer indicator of error related to uncertainty of fine-grain memory. 


\section{Experiment 1}

A

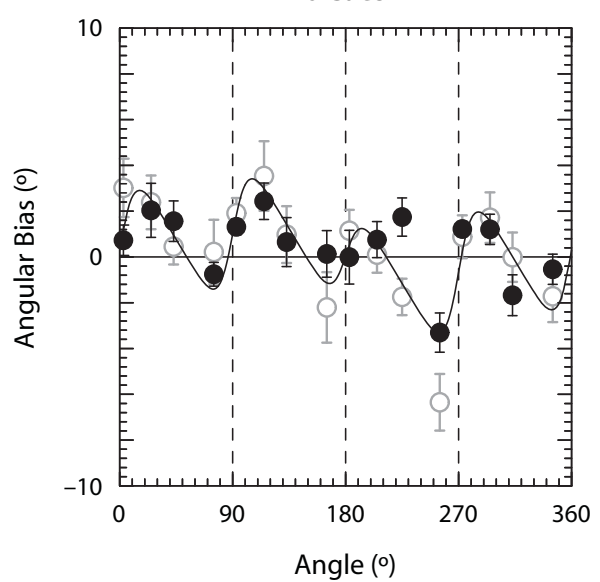

4 Cues

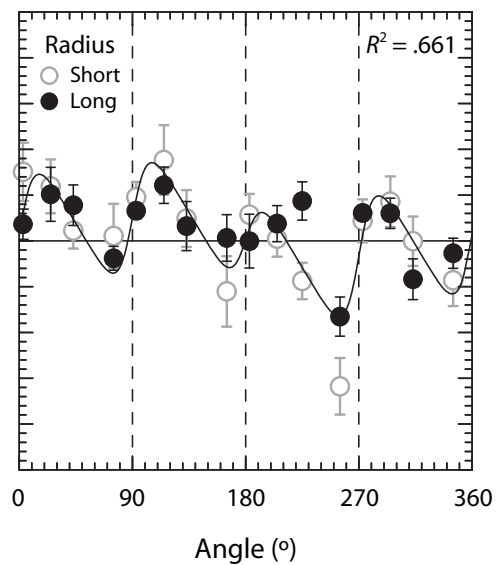

Experiment 2

B

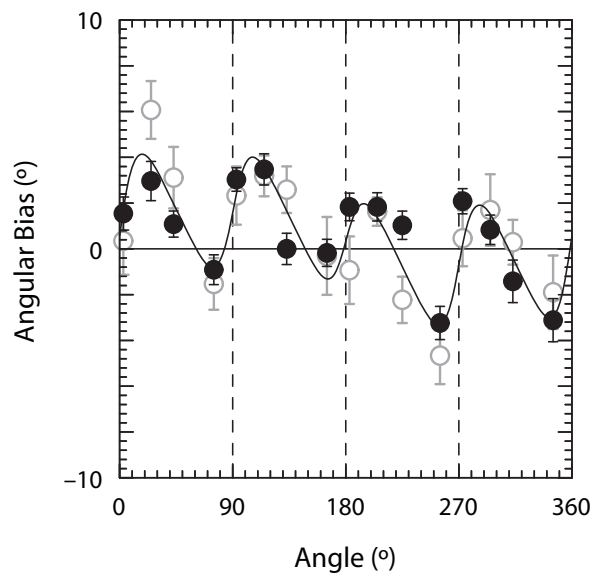

8 Cues

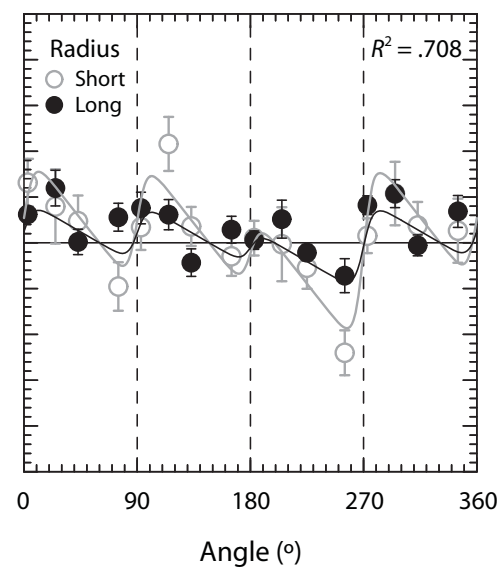

Figure 3. (A) The 6-parameter fixed-quadrants fuzzy-boundary model on angular bias illustrated separately for cue condition in Experiment 1. (B) The 11-parameter fixed-quadrants fuzzyboundary model on angular bias illustrated separate for cue condition in Experiment 2.

As is shown in Table 1, a significant effect for cue was noted, with estimates being more reliable in the 4-cue condition $(M=8.85)$ than in the 0 -cue condition $(M=$ $12.01)$. This result is consistent with the results for absolute error and indicates that this effect is not simply a result of effects on angular or radial bias. Note that the lack of a main effect of radius for this measure suggests that the corresponding main effect of radius for absolute error was likely due to radial bias effects.

\section{Discussion}

In Experiment 1, there were no effects of cue on either bias measure, consistent with participants relying on the default category structure regardless of the presence of external cues. Consequently, the best model describing the angular bias data was a fixed-quadrant fuzzy-boundary model that was fit to the combined 0 -cue and 4-cue data. To examine the explanatory power of this model, an ANOVA was conducted on the residual estimates. This analysis rendered the effect of angle nonsignificant, indicating that the model could account for the basic bias effects. These results provide strong evidence that the default structure was used regardless of the presence of cues.

The pattern of radial bias was consistent with the radial prototype locations being between that of the long- and short-radius targets. Consequently, estimates of location were biased away from the center of the circle for shortradius locations and toward the center for long-radius locations. This pattern is in accordance with that predicted by the category-adjustment model of Huttenlocher et al. (1991). The only other effect on radial bias was a main effect of angle. Our analysis showed that this was due to an 
overall tendency to locate the dot to the left of its actual location, which could be a motor bias associated with the use of the mouse pointing device, and is not of particular interest here.

Although bias patterns were unaffected by the cue manipulation, we did find an effect of cue condition on both absolute error and inconsistency of estimation, indicating greater accuracy when cues were present. This pattern of results is consistent with the idea that cues may enhance finegrain memory. Because the main effect of radius occurred for absolute error but not for inconsistency of estimation, it seems likely that this effect was related to the differential bias for short and long radii and was not due to differences in fine-grain memory for the two types of targets. The fact that cues reduced inconsistency of estimation without altering bias is consistent with other reports of the independence of these measures (see Spencer \& Hund, 2002, who reported effects of age on variable error but not on spatial drift).

Collectively across dependent measures, Experiment 1 provides a clear, conceptual replication of the lack of cue effects on bias that we reported in our previous work (Fitting et al., 2007). This replication enhances our previous conclusion, because it is based on a design with greater power due to (1) the within-subjects cue manipulation, (2) the positioning of cues to maximally conflict with the default categorization scheme (hence maximizing the effect size), and (3) the equating of the number of cue-based categories with the default number of categories. Unlike our previous result, however, we provided the evidence that external cues can affect the accuracy of memory that may be attributable to stabilize or anchor the fine-grain memory representation. Experiments 2 and 3 explored this effect further and tested for additional cue-related effects by increasing the number of external cues.

\section{EXPERIMENT 2}

In Experiment 1, competing four-category representations were available, with results indicating that the cuebased categorization scheme was ignored in favor of the cue-independent scheme. In Experiment 2, we increased the number of external reference cues to 8, providing enough available cues to support a more detailed categorization scheme. We hypothesized that an increase in the number of external cues might affect memory for location in two ways. First, because the number of reference cues now exceeds the number of implicit categories, a decrease in bias should result from the use of a cue-based category structure. Thus, from an adaptive memory perspective, the switch to a cue-based categorization scheme based on more than 4 cues should provide the benefit of reduced bias. Second, on the basis of the Experiment 1 finding that available cues enhance the accuracy and consistency of estimation, the presence of 8 cues should lead to an enhancement of fine-grain memory as well, reflected in measures of absolute error and inconsistency of estimation.

\section{Method}

Twenty undergraduate students from the University of South Carolina psychology department participant pool participated in the experiment in exchange for course credits. All materials, procedures, and instructions were the same as those described in Experiment 1, with the exception that either 0 external reference cues or 8 external reference cues were presented along the circular field on a given trial, as shown in panel $\mathrm{C}$ of Figure 2. The external reference cues were eight letters (A, D, G, J, M, P, S, and V) arranged in clockwise order $45^{\circ}$ apart, starting from the top of the circular field.

\section{Results}

Data analyses. Outliers were defined as they were in Experiment 1. The data points replaced in the 0 -cue condition were $3.44 \%$ for the short radius and $3.75 \%$ for the long radius. In the 8 -cue condition, $3.75 \%$ of the data points were replaced for the short radius, and $3.44 \%$ were replaced for the long radius. For each dependent variable, a 2 (cue condition) $\times 2$ (radius) $\times 16$ (angles) withinsubjects factorial ANOVA was conducted; the results are displayed in Table 1 (for Experiment 2).

Angular bias. As is shown in Table 1, and in contrast with Experiment 1, a significant cue $\times$ angle interaction was found, indicating the influence of cues on angular bias. The significant angle effect indicated that the bias differed across angles. Consistent with the fuzzyboundary model, the trend tests of angle within quadrant revealed a prominent linear trend $[F(1,19)=37.5, p<$ $.001]$, a quadratic trend $[F(1,19)=29.6, p<.001]$, and a cubic trend $[F(1,19)=8.7, p<.01]$. The presence of a significant cue $\times$ angle interaction led us to free model parameters across 0 - and 8-cue conditions. In modeling the 64 data points ( 32 for each cue condition), we constrained parameters to be equal across cue conditions, as long as this did not result in a significant drop in $R^{2}$. Once again, the fixed-quadrants fuzzy-boundary model provided a good fit to the data, with 11 fitted parameters and $R^{2}=.71$. Table 2 presents the estimated parameter values, and Figure 3B illustrates the 11-parameter model fit. A three-way within-factorial ANOVA on the residuals

Table 2

Parameter Values and Fit Indices Modeling 0-Cue and

8-Cue Conditions Together for Angular Bias (Experiment 2)

\begin{tabular}{|c|c|c|c|c|c|c|c|c|}
\hline \multicolumn{2}{|c|}{ Condition } & \multicolumn{7}{|c|}{ Parameters } \\
\hline Cues & Radius & $p_{1}$ & $p_{2}$ & $p_{3}$ & $p_{4}$ & $\lambda$ & $c$ & $R^{2}$ \\
\hline 0 & $\begin{array}{l}\text { Short } \\
\text { Long }\end{array}$ & $\begin{array}{c}60.03^{\circ} \\
"\end{array}$ & $\begin{array}{c}147.24^{\circ} \\
"\end{array}$ & $\begin{array}{c}218.33^{\circ} \\
"\end{array}$ & $\begin{array}{c}309.62^{\circ} \\
"\end{array}$ & $\begin{array}{c}.883 \\
"\end{array}$ & $\begin{array}{c}0.076 \\
"\end{array}$ & .708 \\
\hline 8 & $\begin{array}{l}\text { Short } \\
\text { Long }\end{array}$ & " & " & $\begin{array}{c}199.48^{\circ} \\
"\end{array}$ & $\begin{array}{c}330.86^{\circ} \\
"\end{array}$ & $\begin{array}{l}.930 \\
.968 \\
\end{array}$ & $\begin{array}{c}0.131 \\
"\end{array}$ & \\
\hline
\end{tabular}

Note $-p_{1}, p_{2}, p_{3}, p_{4}$, prototype values; $\lambda$, weight of fine-grain memory; $c$, sensitivity parameter. 
for the 11-parameter model revealed the disappearance of all significant effects (see Table 1 for Experiment 2), indicating that the 11-parameter fixed-quadrants fuzzyboundary model provided a good fit to the empirical data. The parameter values shown in Table 2 for the 0 -cue condition were very close to those found in Experiment 1. An adequate fit to the 8-cue data required fitting additional values of the fuzzy-boundary parameter, $c$, fine-grain weighting for long and short radii, $\lambda_{\mathrm{L}}$ and $\lambda_{\mathrm{S}}$, and slight variations in the locations of prototypes in the third and fourth quadrant $\left(p_{3}, p_{4}\right)$. A comparison of model parameters across cue conditions indicates that including 8 cues increased the weighting of fine-grain memory values, especially for the long-radius locations (i.e., nearest to the cues). However, the key finding here was that the fourquadrant representation adequately described the 8-cue condition, indicating no effect of cues on the structuring of categories that bias estimates.

Radial bias. As is shown in Table 1, all three main effects were significant for radial bias. The main effect of radius was consistent with a radial prototype between short and long-radius targets $\left(M_{\text {short }}=8.78\right.$ and $\left.M_{\text {long }}=-1.76\right)$. The main effect of cue condition was consistent with the radial prototype being located more toward the circumference in the 8-cue condition $\left(M_{0}=2.67\right.$ and $\left.M_{8}=4.15\right)$. Finally, the main effect of angle appeared to be primarily due to a leftward bias in estimation that has been observed elsewhere (Fitting et al., 2007; Wedell et al., 2007).

Absolute error and inconsistency of estimation. As is shown in Table 1, the ANOVA on absolute error revealed significant main effects of cue $\left(M_{0}=15.70\right.$ and $\left.M_{8}=14.54\right)$ and radius $\left(M_{\text {short }}=17.40\right.$ and $M_{\text {long }}=$ 12.84 ), along with a significant cue $\times$ radius interaction. The interaction reflected the fact that the 8-cue condition increased accuracy for the long radius $\left(M_{8-\text { long }}=11.60\right)$ but that it did not influence accuracy for the short radius $\left(M_{8 \text {-short }}=17.47\right)$. No difference was noted for the 0 -cue condition between the short radius $\left(M_{0-\text { short }}=17.33\right)$ or the long radius $\left(M_{0-\text { long }}=14.07\right)$. In addition, a significant effect for angle was noted.

Table 1 also shows the results of the ANOVA on inconsistency of estimation. Significant main effects for cue and radius were noted, with no significant interaction and no significant main effect of angle. Inconsistency of estimation was lower in the 8-cue condition $\left(M_{0}=10.62\right.$ and $\left.M_{3}=8.93\right)$ and for the long-radius targets $\left(M_{\text {short }}=11.40\right.$ and $M_{\text {long }}=8.16$ ). The lack of an interaction between cue and radius implies that the corresponding interaction for absolute error may have resulted from the radial bias effect. As the radial prototype moved outward in the 8-cue condition, it produced proportionately greater bias, contributing to greater error for the short-radius targets.

\section{Discussion}

Unlike results from our previous research (Fitting et al., 2007) and from Experiment 1, both angular and radial biases were affected by the number of cues in Experiment 2. The significant cue effect on radial bias indicates that the radial prototype was shifted outward with 8 cues. An outer perimeter of cues might have led participants to perceive an extended task field. Wedell et al. (2007) demonstrated that prototypes tend to be shifted outward with extension of the task field, even if target locations are unchanged. This apparently did not happen with 1,3 , or 4 cues, perhaps because these cues were not spaced closely enough together to create the perception of an extended circular task field. This cue-related extension may have caused the radial prototype - typically located between short- and long-radius targets - to shift beyond the long radius targets.

The cue $\times$ angle interaction on angular bias demonstrated a second way in which cues affected bias. This interaction did not reflect a restructuring of the categories with the presence of cues. Rather, cues appear to have enhanced fine-grain memory, especially for long-radius targets. Thus, the slopes of the bias functions were reduced in the 8-cue condition rather than the number of inferred prototypes being changed or the locations of the inferred prototypes being shifted drastically. These conclusions are supported by the good fit of the fixed-quadrants fuzzy-boundary model. The fine-grain memory was much greater for the 8-cue condition than for the 0 -cue condition. This can be seen in the reduced slopes for the 8-cue condition (Figure 3B) and also by the increased inferred values for this parameter from the model fits (the $\lambda \mathrm{s}$ in Table 2). Only one $\lambda$ was necessary to fit the empirical data in the 0 -cue condition, whereas two $\lambda$ values were needed in the 8-cue condition, indicating greater fine-grain memory weighting for the long-radius targets located closer to the 8 external cues.

The results from analyzing absolute error and inconsistency of estimation measures generally support the conclusions drawn above. Both of these measures were reduced for long versus short radius and for 8 versus 0 cues. Of particular interest was the effect of cues on these measures. As in Experiment 1, the surrounding cues appear to have provided a way to hold fine-grain values in memory and hence produce more consistent and accurate estimates.

In summary, Experiment 2 replicated the key finding from Experiment 1 that the fixed-quadrants fuzzyboundary model explains most of the variance of the data. This result supports the idea that a cue-independent frame of reference is used to generate the category structure in the static environment, despite the abundant presence of cues. Further, results from Experiment 2 reinforced results from Experiment 1, indicating that cues may serve to anchor memory for location, reflected in significant main effects of cue for absolute error and inconsistency of estimation. Thus, given sufficient numbers of cues, finegrain memory may be enhanced by their presence. Finally, results from Experiment 2 were consistent with the idea that, given enough surrounding cues, the perceptual field may be enlarged and lead to a more extreme radial prototype, as indicated by the cue effect on radial bias.

\section{EXPERIMENT 3}

Experiments 1 and 2 demonstrated that in a static task field, the available reference cues (4 or 8, respectively) were not used to generate categories for encoding locations. Instead, memory was biased by default categories. Although restructuring of categorical memory was not 
evident in Experiment 1, both experiments provided evidence that external cues may result in reduced absolute error and inconsistency of estimation. The goal of Experiment 3 was twofold. First, we increased the number of surrounding cues to 24 to prompt structural changes and further test for cue effects on fine-grain memory. Second, we were interested in how the timing conditions would affect fine-grain memory. We hypothesized that an increase in the presentation time of the dot location may lead to increased encoding time and hence potentially increase the accuracy of memory for the object location. Furthermore, increased encoding time might facilitate the use of the available external reference cues and potentially change the category structure. For delay time, we hypothesized that the bias and error effects should be magnified with a decrease in fine-grain memory associated with greatest delay (Engebretson \& Huttenlocher, 1996; Hund \& Plumert, 2002; Spencer \& Hund, 2003). For the combination of increased presentation time with increased delay time, we hypothesized that cues might be more readily encoded and used, resulting in the greatest reliance on the available external reference cues.

\section{Method}

Ninety undergraduate students participated in the experiment in exchange for course credits. All aspects of the materials, procedure, and instructions were the same as those in Experiments 1 and 2, except for the number of cues and the timing of stimulus presentation and delay. Cue was manipulated by presenting either 0 or 24 external reference cues along the circular region. The external reference cues were letters ranging from $\mathrm{A}$ to $\mathrm{X}$ in a clockwise order spaced $15^{\circ}$ apart, as shown in panel D of Figure 2. Timing conditions were manipulated between subjects, with the four conditions resulting from the $2 \times 2$ combination of presentation time ( 1 or $3 \mathrm{sec}$ ) and delay time $(1.5$ or $4.5 \mathrm{sec})$. All conditions had 20 participants, except for the combination of the 1 -sec presentation and the 4.5 -sec delay, which had 30 participants.

\section{Results}

Data analyses. Outliers were defined as they were in Experiments 1 and 2 . In the 0 -cue condition across radius, presentation, and delay conditions, an average of $3.60 \%$ were replaced, ranging from $3.13 \%-3.75 \%$. In the $24-$ cue condition, the average was lower, with $2.11 \%$ ranging from $1.56 \%-2.50 \%$. For each of the four dependent variables, a 2 (presentation time) $\times 2$ (delay time) $\times 2$ (cue condition) $\times 2$ (radius) $\times 16$ (angle) mixed-factorial ANOVA was conducted; Table 3 summarizes the results.

Angular bias. As is shown in Table 3, and consistent with results from Experiment 2, there was a significant cue $X$ angle interaction, indicating that cues affected the pattern of angular bias. The other significant effects on angular bias included a main effect of cue, a main effect of angle, a cue $\times$ presentation interaction, and a radius $\times$

Table 3

Degrees of Freedom $(d f)$ and $F$ Values for 2 (Present) $\times 2$ (Delay) $\times 2$ (Cue Condition) $\times$ 2 (Radius) $\times 16$ (Actual Angle) Mixed Factorial ANOVAs of Experimment 3

\begin{tabular}{|c|c|c|c|c|c|c|c|}
\hline Source & $d f$ & $\begin{array}{l}\text { Angular } \\
\text { Bias }\end{array}$ & $\begin{array}{c}\text { Residuals 16P } \\
\text { FQFBM }\end{array}$ & $\begin{array}{l}\text { Residuals 13P } \\
\text { FQFBM }\end{array}$ & $\begin{array}{l}\text { Absolute } \\
\text { Error }\end{array}$ & $\begin{array}{c}\text { Inconsistency } \\
\text { of Estimate }\end{array}$ & $\begin{array}{l}\text { Radial } \\
\text { Bias }\end{array}$ \\
\hline Presentation (P) & $(1,86)$ & 0.43 & 0.06 & 0.00 & $8.13^{* *}$ & $5.95^{*}$ & $5.64^{*}$ \\
\hline Delay (D) & $(1,86)$ & 0.29 & 0.05 & 0.00 & $41.81^{* * *}$ & $26.89^{* * *}$ & $16.59^{* * * *}$ \\
\hline $\mathrm{P} \times \mathrm{D}$ & $(1,86)$ & 0.95 & 0.01 & 0.00 & 0.85 & 1.24 & 0.31 \\
\hline Cue $(\mathrm{C})$ & $(1,86)$ & $5.38^{*}$ & 0.10 & 0.00 & 1.27 & $6.05^{*}$ & $54.93^{* * *}$ \\
\hline $\mathrm{C} \times \mathrm{P}$ & $(1,86)$ & $4.25^{*}$ & 0.11 & 0.00 & 0.14 & 2.81 & 2.92 \\
\hline $\mathrm{C} \times \mathrm{D}$ & $(1,86)$ & 0.10 & 0.01 & 0.00 & $4.76^{*}$ & 3.17 & $6.06^{*}$ \\
\hline $\mathrm{C} \times \mathrm{P} \times \mathrm{D}$ & $(1,86)$ & 3.44 & 0.03 & 0.00 & 0.43 & 0.31 & $5.57^{*}$ \\
\hline Radius (R) & $(1,86)$ & 0.26 & 0.31 & 0.16 & $95.23^{* * *}$ & $79.23^{* * *}$ & $116.98^{* * *}$ \\
\hline $\mathrm{R} \times \mathrm{P}$ & $(3,86)$ & 2.34 & 0.60 & 2.48 & 0.67 & $4.82^{*}$ & 1.41 \\
\hline $\mathrm{R} \times \mathrm{D}$ & $(3,86)$ & 0.85 & 1.53 & 0.86 & $40.93^{* * * *}$ & $27.41^{* * * *}$ & $38.20^{* * *}$ \\
\hline $\mathrm{R} \times \mathrm{P} \times \mathrm{D}$ & $(3,86)$ & 0.00 & 0.22 & 0.01 & 1.75 & 0.31 & 3.29 \\
\hline Angle (A) & $(15,1290)$ & $21.68^{* * *}$ & $3.20^{* *}$ & $3.32^{* *}$ & $3.42^{* * *}$ & 1.52 & $13.70^{* * *}$ \\
\hline $\mathrm{A} \times \mathrm{P}$ & $(15,1290)$ & 1.60 & 0.64 & 0.63 & 0.29 & 0.80 & 0.98 \\
\hline$A \times D$ & $(15,1290)$ & 1.57 & 0.57 & 0.60 & 1.23 & 0.66 & $2.13^{* *}$ \\
\hline $\mathrm{A} \times \mathrm{P} \times \mathrm{D}$ & $(15,1290)$ & 0.88 & 0.39 & 0.38 & 0.64 & 0.46 & 1.16 \\
\hline $\mathrm{C} \times \mathrm{R}$ & $(1,86)$ & 1.08 & 0.54 & 1.29 & $61.31^{* * * *}$ & $40.03^{* * *}$ & 2.00 \\
\hline $\mathrm{C} \times \mathrm{R} \times \mathrm{P}$ & $(1,86)$ & 0.95 & 0.58 & 1.03 & 2.03 & 0.00 & 0.13 \\
\hline $\mathrm{C} \times \mathrm{R} \times \mathrm{D}$ & $(1,86)$ & 0.10 & 0.08 & 0.10 & $8.86^{* *}$ & $8.04^{* *}$ & 2.31 \\
\hline $\mathrm{C} \times \mathrm{R} \times \mathrm{P} \times \mathrm{D}$ & $(1,86)$ & 0.41 & 0.01 & 0.36 & 0.15 & 0.04 & 1.44 \\
\hline $\mathrm{C} \times \mathrm{A}$ & $(15,1290)$ & $6.87^{* * * *}$ & 1.48 & 1.41 & 1.15 & 0.77 & 1.12 \\
\hline $\mathrm{C} \times \mathrm{A} \times \mathrm{P}$ & $(15,1290)$ & 0.53 & 0.20 & 0.21 & 1.02 & 1.30 & 1.10 \\
\hline $\mathrm{C} \times \mathrm{A} \times \mathrm{D}$ & $(15,1290)$ & 1.28 & 0.43 & 0.43 & 0.55 & 0.55 & 0.53 \\
\hline $\mathrm{C} \times \mathrm{A} \times \mathrm{P} \times \mathrm{D}$ & $(15,1290)$ & 0.95 & 0.61 & 0.63 & 1.22 & 1.30 & 0.80 \\
\hline $\mathrm{R} \times \mathrm{A}$ & $(15,1290)$ & $4.42^{* * * *}$ & $2.60^{* *}$ & $3.03^{* * * *}$ & $2.00^{*}$ & 0.49 & $2.61^{* *}$ \\
\hline $\mathrm{R} \times \mathrm{A} \times \mathrm{P}$ & $(15,1290)$ & 0.86 & 0.72 & 0.82 & 0.65 & 0.86 & 0.68 \\
\hline $\mathrm{R} \times \mathrm{A} \times \mathrm{D}$ & $(15,1290)$ & 0.65 & 0.65 & 0.62 & $2.25^{* *}$ & 0.64 & $2.58^{* *}$ \\
\hline $\mathrm{R} \times \mathrm{A} \times \mathrm{P} \times \mathrm{D}$ & $(15,1290)$ & 0.71 & 0.72 & 0.73 & 1.04 & 0.62 & 1.28 \\
\hline $\mathrm{C} \times \mathrm{R} \times \mathrm{A}$ & $(15,1290)$ & 0.82 & 0.72 & 1.88 & 0.84 & 1.52 & 1.10 \\
\hline $\mathrm{C} \times \mathrm{R} \times \mathrm{A} \times \mathrm{P}$ & $(15,1290)$ & 1.05 & 0.80 & 0.96 & 0.82 & 1.10 & 0.36 \\
\hline $\mathrm{C} \times \mathrm{R} \times \mathrm{A} \times \mathrm{D}$ & $(15,1290)$ & 1.20 & 0.86 & 1.18 & 0.69 & 0.92 & 0.55 \\
\hline $\mathrm{C} \times \mathrm{R} \times \mathrm{A} \times \mathrm{P} \times \mathrm{D}$ & $(15,1290)$ & 1.08 & 0.74 & 0.87 & 0.67 & 0.41 & 0.90 \\
\hline
\end{tabular}


Table 4

Parameter Values and Fit Indices Modeling 0-Cue and 24-Cue Conditions Across Timing Manipulation for Angular Bias (Experiment 3)

\begin{tabular}{|c|c|c|c|c|c|c|c|c|c|}
\hline \multicolumn{3}{|c|}{ Condition } & \multicolumn{7}{|c|}{ Parameters } \\
\hline Timing & Cues & Radius & $p_{1}$ & $p_{2}$ & $p_{3}$ & $p_{4}$ & $\lambda$ & $c$ & $R^{2}$ \\
\hline \multirow[t]{2}{*}{$\begin{array}{l}\text { 1-sec presentation, } \\
1.5 \text {-sec delay }\end{array}$} & 0 & $\begin{array}{l}\text { Short } \\
\text { Long }\end{array}$ & 53.11 & $\begin{array}{c}143.32 \\
"\end{array}$ & $\begin{array}{c}222.60 \\
"\end{array}$ & $\begin{array}{c}309.82 \\
" 1\end{array}$ & $\begin{array}{c}904 \\
"\end{array}$ & $\begin{array}{c}0.046 \\
"\end{array}$ & .654 \\
\hline & 24 & $\begin{array}{l}\text { Short } \\
\text { Long }\end{array}$ & $\begin{array}{l}39.23 \\
"\end{array}$ & $\begin{array}{c}146.43 \\
"\end{array}$ & $\begin{array}{c}186.38 \\
"\end{array}$ & $\begin{array}{c}316.48 \\
"\end{array}$ & $\begin{array}{c}.962 \\
" ~\end{array}$ & $\begin{array}{l}0.112 \\
0.041\end{array}$ & \\
\hline \multirow[t]{2}{*}{$\begin{array}{l}\text { 1-sec presentation, } \\
\text { 4.5-sec delay }\end{array}$} & 0 & $\begin{array}{l}\text { Short } \\
\text { Long }\end{array}$ & $\begin{array}{c}45.75 \\
"\end{array}$ & $\begin{array}{c}147.12 \\
"\end{array}$ & $\begin{array}{c}226.21 \\
" ~\end{array}$ & $\begin{array}{c}310.23 \\
"\end{array}$ & $\begin{array}{c}.894 \\
" ~\end{array}$ & $\begin{array}{c}0.090 \\
"\end{array}$ & .744 \\
\hline & 24 & $\begin{array}{l}\text { Short } \\
\text { Long }\end{array}$ & $\begin{array}{c}45.99 \\
"\end{array}$ & $\begin{array}{c}134.76 \\
"\end{array}$ & $\begin{array}{c}210.57 \\
"\end{array}$ & $\begin{array}{c}325.63 \\
"\end{array}$ & $\begin{array}{c}.901 \\
"\end{array}$ & $\begin{array}{l}0.061 \\
0.030\end{array}$ & \\
\hline \multirow[t]{2}{*}{$\begin{array}{l}\text { 3-sec presentation, } \\
1.5 \text {-sec delay }\end{array}$} & 0 & $\begin{array}{l}\text { Short } \\
\text { Long }\end{array}$ & $\begin{array}{c}60.01 \\
"\end{array}$ & $\begin{array}{c}141.41 \\
"\end{array}$ & $\begin{array}{c}221.39 \\
"\end{array}$ & $\begin{array}{c}307.02 \\
"\end{array}$ & $\begin{array}{c}.946 \\
" ~\end{array}$ & $\begin{array}{c}0.089 \\
"\end{array}$ & .644 \\
\hline & 24 & $\begin{array}{l}\text { Short } \\
\text { Long }\end{array}$ & $\begin{array}{c}64.34 \\
"\end{array}$ & $\begin{array}{c}138.57 \\
"\end{array}$ & $\begin{array}{c}208.46 \\
"\end{array}$ & $\begin{array}{c}330.36 \\
"\end{array}$ & $\begin{array}{c}.955 \\
" ~\end{array}$ & $\begin{array}{l}0.052 \\
0.024\end{array}$ & \\
\hline \multirow[t]{2}{*}{$\begin{array}{l}\text { 3-sec presentation, } \\
4.5 \text {-sec delay }\end{array}$} & 0 & $\begin{array}{l}\text { Short } \\
\text { Long }\end{array}$ & $\begin{array}{l}50.33 \\
"\end{array}$ & $\begin{array}{c}151.16 \\
"\end{array}$ & $\begin{array}{c}207.28 \\
"\end{array}$ & $\begin{array}{c}318.97 \\
"\end{array}$ & $\begin{array}{c}.942 \\
"\end{array}$ & 0.155 & .600 \\
\hline & 24 & $\begin{array}{l}\text { Short } \\
\text { Long }\end{array}$ & $\begin{array}{c}69.99 \\
" ~\end{array}$ & $\begin{array}{c}136.02 \\
"\end{array}$ & $\begin{array}{c}184.96 \\
"\end{array}$ & $\begin{array}{c}331.35 \\
"\end{array}$ & $\begin{array}{c}.971 \\
"\end{array}$ & $\begin{array}{l}0.081 \\
0.041\end{array}$ & \\
\hline
\end{tabular}

Note $-p_{1}, p_{2}, p_{3}, p_{4}$, prototype values; $\lambda$, weight of fine-grain memory; $c$, sensitivity parameter.

angle interaction. In a more fine-grain analysis of the data, we conducted a six-way mixed ANOVA that examined the effects of angle within each quadrant. This analysis demonstrated, among other things, significant effects of angle $\times$ presentation $\left[F(3,258)=5.9, p_{\mathrm{GG}}<.01\right]$ and of angle $\times$ delay $\left[F(3,258)=5.3, p_{\mathrm{GG}}<.01\right]$. These interaction effects justify presenting and modeling these conditions separately.

Table 4 presents the estimated parameter values from the fixed-quadrants fuzzy-boundary modeling of the data, and Figure 4 illustrates the angular bias data along with the model fits to the four timing conditions. To facilitate comparisons across the four timing conditions of Experiment 3, we examined different ways to apply parallel constraints across conditions. After trying several different approaches, we determined that a 13-parameter fit to the 64 data points within each timing condition provided the most parsimonious description across conditions. Our model presentation in this section is geared to provide a model-based comparison across conditions rather than a rigorous test of different versions of the model. We used the same 6-parameter model to fit the 0 -cue conditions as used in Experiments 1 and 2, with one value of fine-grain weighting $(\lambda)$, four prototype values $\left(p_{1}, p_{2}, p_{3}, p_{4}\right)$, and one value of the boundary sensitivity parameter $(c)$. The same 6 parameters were fit to the 24-cue condition, with the addition of a separate boundary sensitivity parameter for long-radius targets. This parameter was added primarily to explain differences in the angular bias effects for long- and shortradius targets in the 24-cue condition. The 13-parameter fixed-quadrants fuzzy-boundary model provided reasonably good fits to the data in each timing condition, with the $R^{2}$ values shown in Figure 4. A five-way mixed factorial ANOVA on the residuals for the 13-parameter models revealed the disappearance of all significant effects, except for the angle effect and the angle $\times$ radius interaction (see Table 3). However, note that the angle effect was reduced from an $F$ value of 21.7 to an $F$ value of 3.3, indicating that the 13-parameter fixed-quadrants fuzzy-boundary model explained most of the relevant variance in the empirical data.

In examining the model fit for the 0 -cue conditions first, as shown in Table 4, it is clear that the four prototypes tend to be centrally located within quadrant in each case. The fine-grain weighting parameter, $\lambda$, appears to be sensitive to encoding time, but not to delay time. Instead, increased delay leads to higher categorical sensitivity expressed in the $c$ parameter. The fit of the model shown in the left panels of Figure 4 illustrates the regularity of the data. The increased sensitivity parameter leads to steeper sloped bias functions for the 4.5 -sec delay conditions, suggesting a greater reliance on the categorical representation. As a point of reference, the $1-\mathrm{sec}$ presentation and the 4.5 -sec delay condition are the same as those used in Experiments 1 and 2 (Figures $3 \mathrm{~A}$ and $3 \mathrm{~B}$ ), and the pattern of data is very similar. When encoding time is increased, the magnitude of bias is decreased, as illustrated in the reduced slopes in the corresponding graphs of Figure 4.

A comparison of prototype locations suggests that these are fairly similar in the 0 -cue and 24-cue conditions. The main exception appears to be in the third quadrant, where two of the inferred prototypes for 24-cue conditions lie very near the border $\left(180^{\circ}\right)$. Although there is a fairly wide variation of prototype locations within quadrants reported in the literature, it may be that the 24-cue condition affords more flexibility of locating prototypes within a quadrant. Beyond this slightly more idiosyncratic location of prototypes, a clear effect of cues can be seen in the fine-grain weighting parameter, $\lambda$. Although the differences are fairly small, the fine-grain weighting value is higher for the 24cue condition than for the corresponding 0 -cue condition. This is consistent with the idea that cues serve to anchor fine-grain memory values. The other consistent finding in the 24-cue condition is that the sensitivity parameter is greater for the short than for the long-radius targets within 
0 Cues

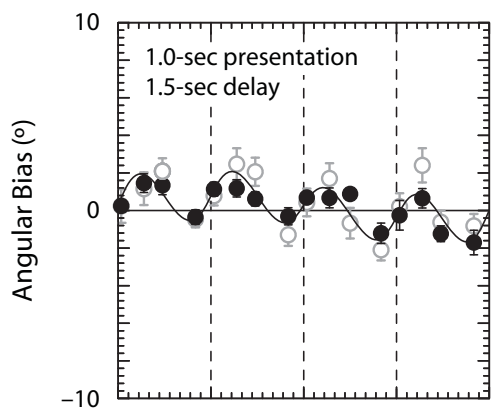

0 Cues

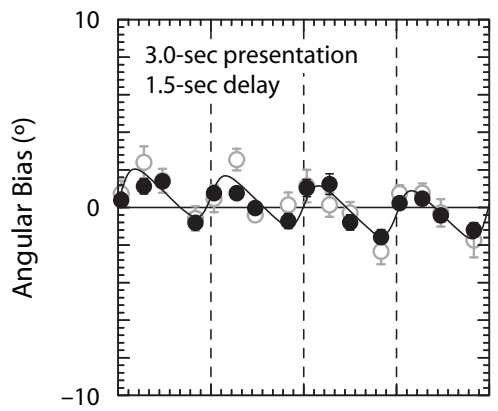

0 Cues

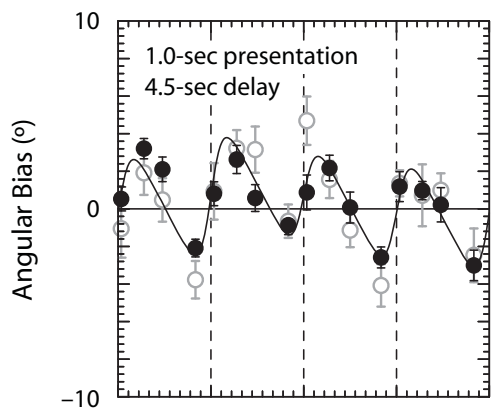

0 Cues

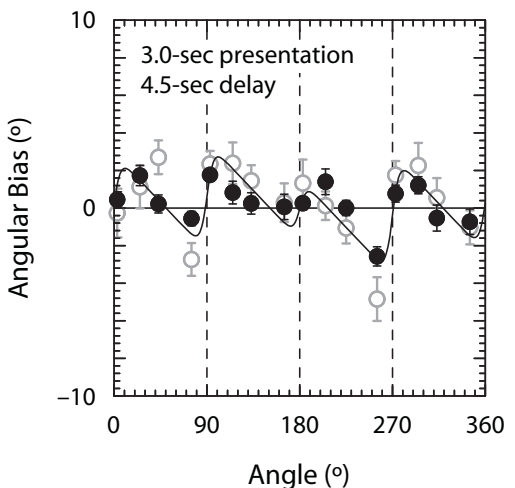

24 Cues

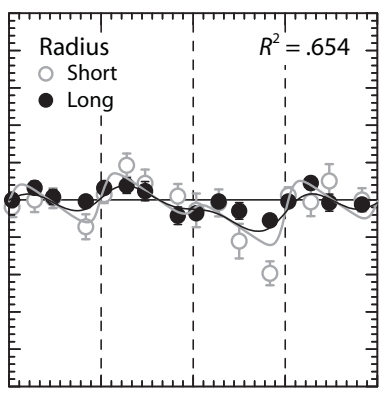

24 Cues

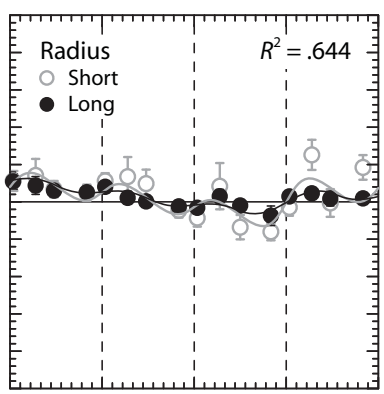

24 Cues

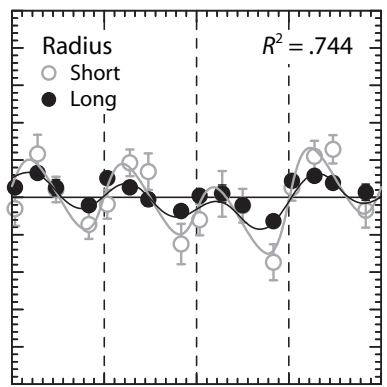

24 Cues

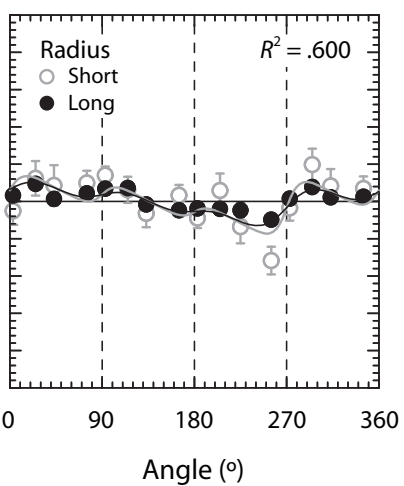

Figure 4. The 13-parameter fixed-quadrants fuzzy-boundary model on angular bias (one $\lambda$ and one $c$ for the 0 -cue condition, whereas one $\lambda$ but two cs separate for radius were fitted in the 24-cue condition) presented by radius, timing manipulation, and cue condition (Experiment 3). 
each timing condition. This suggests a greater adherence to categorical processing for the short-radius targets.

Radial bias. Figure 5 illustrates the mean radial bias as a function of cue and radius for each of the four timing manipulations. As is shown in Table 3, significant main effects were noted for cue and radius, with the radial bias being driven toward the circumference more so for the 24-cue condition $(M=4.31)$ than for the 0 -cue condition $(M=$ $1.75)$ and more so for the short radius $(M=6.63)$ than for the long radius $(M=-0.57)$. Cue effects were significantly altered by delay time (cue $\times$ delay interaction), indicating that an increase in delay time resulted in a greater shift of the radial prototype toward the perimeter when cues were available. However, the three-way interaction of cue, presentation, and delay indicated that the above two-way interaction was limited to the $1-\mathrm{sec}$ presentation condition.

Absolute error and inconsistency of estimation. Table 3 shows that for absolute error, there was a significant cue $\times$ radius interaction, with the presence of 24 cues reducing absolute error much more for the long-radius than for the short-radius targets. A cue $\times$ delay interaction indicated that absolute error was more strongly reduced in the 24-cue condition at the increased delay. The radius $\times$ delay interaction indicated that the absolute error advantage of the long-radius targets was much greater at the increased delay. Finally, the cue $X$ radius $X$ delay interaction reflected the stronger cue $X$ radius interaction at the 4.5 -sec delay. The ANOVA results for inconsistency of estimation (for the most part) parallel those for absolute error. Although there are a few differences in the patterns of significance, the inconsistency of estimation data supports the general conclusions drawn from the absolute error data that fine-grain memory increases with the presence of cues, more time for encoding, and decrease in delay. These results also support the conclusion that cue effects on fine-grain memory are greater for the locations more proximal to the cues (i.e., the longer radius targets). Given the parallel results, we provide only an illustration of the results for inconsistency of estimation in Figure 6.

\section{A}

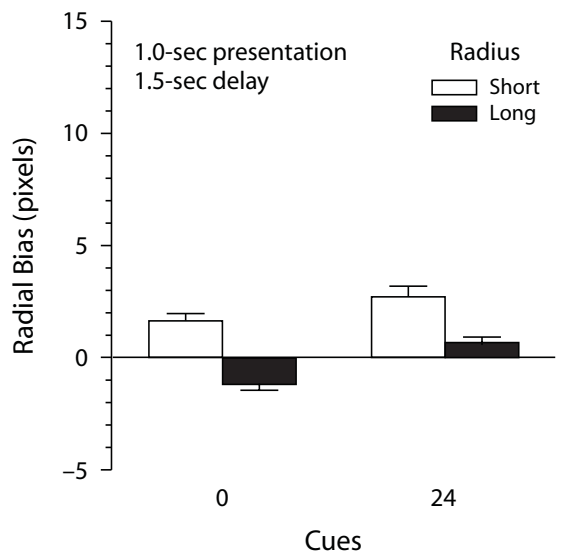

C

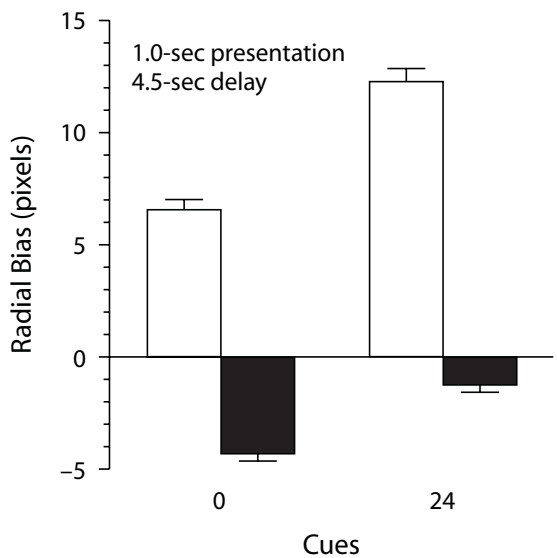

B

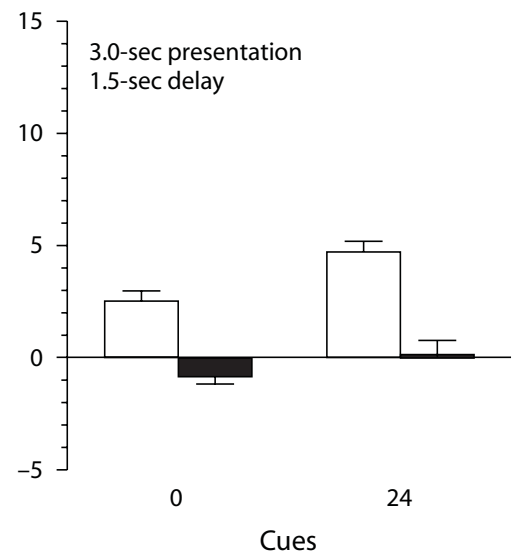

D

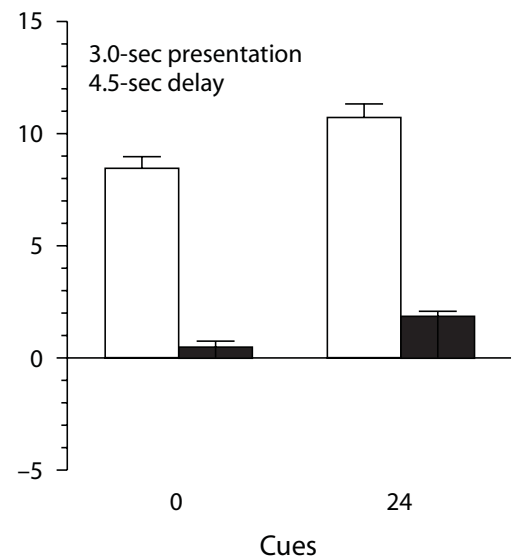

Figure 5. Mean radial bias scores for the short and long radius separate for timing and cue condition. The error bars indicate one standard error of the mean (Experiment 3). 
A

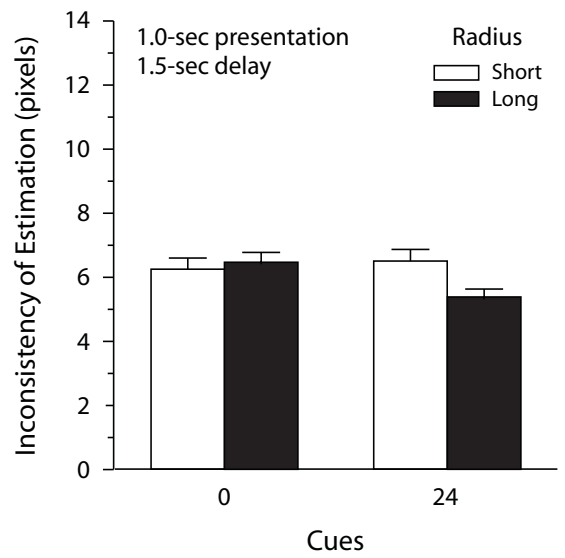

\section{C}

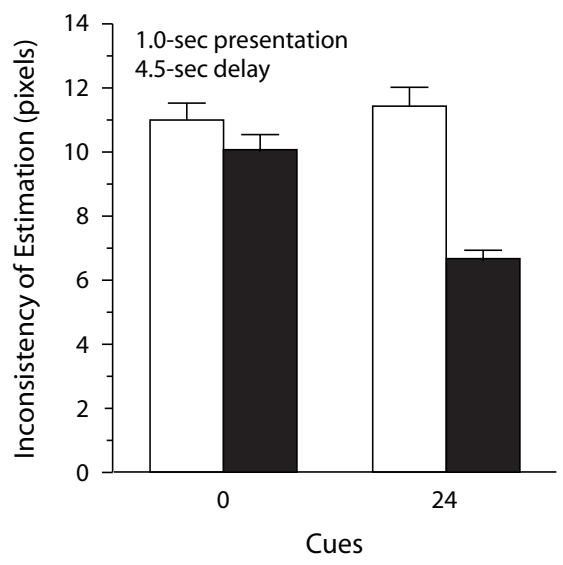

B

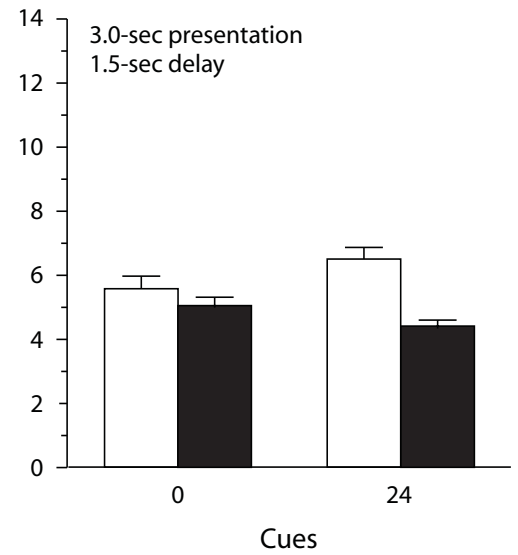

D

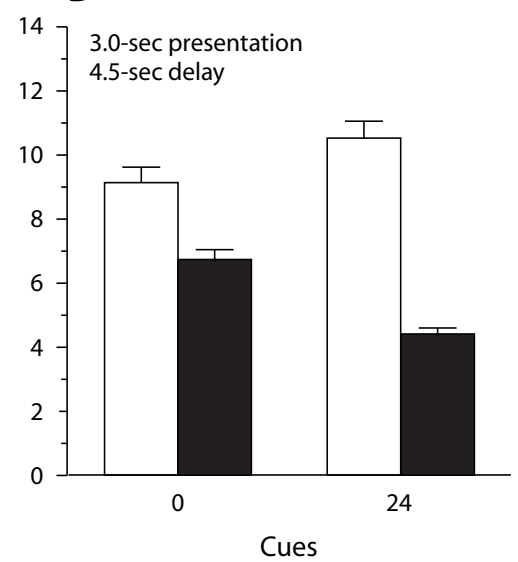

Figure 6. Mean inconsistency of estimation measure. The error bars indicate one standard error of the mean (Experiment 3).

\section{Discussion}

As in Experiments 1 and 2, the available reference cues in Experiment 3 did not result in a categorization scheme radically different from the default scheme used when no cues were available. Thus, Experiments 1-3 provide strong evidence that across a wide number of available cues, cue locations, and timing procedures, participants use the same four-quadrant categorization scheme to organize spatial memory.

Implicit categories produced less angular bias when 24 cues were available, especially for the proximal (long-radius) targets. These findings are illustrated in the different slopes in Figure 4 and corresponding changes in fine-grain memory $(\lambda)$ and sensitivity $(c)$ in Table 4 . This result is consistent with that reported for the 8-cue condition of Experiment 2. Results on absolute error and inconsistency of estimation provided further support for the idea that the external cues anchor fine-grain memory for proximal targets.

An objection that might be raised against our methodology is that interspersing cue trials and 0 -cue trials might lead participants to adopt a single categorization scheme for both conditions. We do not believe that this was the case. In an additional experiment, we blocked the presentation of the 24-cue trials together and followed this by a blocked presentation of the 0 -cue trials (presentation, $1 \mathrm{sec}$; delay, $1.5 \mathrm{sec} ; N=20$ ). The results were nearly identical to those shown in Figure 4 for the corresponding conditions. Because participants continued to use the four-quadrant categorization scheme even when the 24-cue trials were blocked together and they had no prior experiences with the 0 -cue trials, we believe that the categorization scheme is not an artifact of our method.

Experiment 3 results provided additional insights into the role of presentation time and delay time and supported findings by other researchers about the dynamics of spatial memory over time (Hund \& Plumert, 2002; Simmering et al., 2006; Spencer et al., 2006). Presentation time effects on error measures were consistent with the idea that increased encoding time helped to increase fine-grain memory stability, which was modeled for the angular bias data as increased weighting of fine-grain memory. Estimation of a briefly presented dot location with an ex- 
tended time delay before retrieval was, as expected, the least accurate of the four conditions. However, the 24-cue long-radius targets were least affected by the brief encoding and extended forgetting time, presumably due to cues anchoring fine-grain memory for proximal targets.

As was expected, increasing delay resulted in poorer fine-grain memory, reflected by increased absolute error and inconsistency of estimation. Consequently, increased delay resulted in greater reliance on the categorical representation, as illustrated by the steeper slopes for angular bias (Figure 4) and modeled as sharper boundary sensitivity. The cue $\times$ radius $\times$ delay interaction for both absolute error and inconsistency of estimation implicates cues as a mechanism to buffer the effects of delay for proximal targets. Although increasing delay led to large increases in absolute error and inconsistency of estimation in the 0 -cue condition, these increases were limited to the short-radius targets in the 24-cue condition. Thus, proximal cues appear to anchor fine-grain memory for long-radius targets, stabilizing the representation so it was less degraded across time.

\section{EXPERIMENT 4}

In Experiments 1-3, we demonstrated that in a static task field, available reference cues (varying from 4 to 24) were not used to create a categorization scheme that was qualitatively different from the default scheme used when no cues were available. Although providing 8 or 24 cues produced less angular bias in Experiments 2 and 3, all three experiments provide strong evidence that participants use the same four-quadrant categorization scheme to organize spatial memory regardless of cue availability. Thus, although cues do not change the categorization scheme, they do appear to anchor fine-grain memory and thereby reduce absolute error and inconsistency of estimation.

In Experiment 4, we examined the question of whether these results hold when memory for target location is more severely disrupted. On the basis of our extensive pilot testing of different procedures, we disrupted memory with two key manipulations. First, instead of presenting one target followed by a mask, we presented sequentially four targets, each followed by a mask. Each target dot was presented in a different color so that we could prompt the participant with a given color to elicit an estimation of that dot's location. Presenting successively four different dot locations presumably loaded working memory and also increased memory interference. Second, after all four targets were presented, participants were asked to solve a Shepard-Metzler mental rotation task for at least $10 \mathrm{sec}$. This task mentally engaged spatial working memory resources, once again making it difficult to hold the finegrain memory locations in memory and thereby increasing reliance on categorical memory.

In Experiment 4, we manipulated cues between subjects so that participants had either 0 or 4 cues at locations identical to the one used in Experiment 1 (shown in panel B of Figure 2). In the 0-cue condition, we anticipated that participants would once again rely on the default category structure found in Experiments 1-3. However, if cues are considered useful for coding locations under memory- taxing conditions, then we would expect those participants in the 4-cue condition to adopt a cue-based category structure. Because this structure was rotated $45^{\circ}$ relative to the default structure, we expected to be able to detect a very different pattern of angular bias, if indeed the cues were being used in this way.

\section{Method}

Eighty-four undergraduate students participated in the experiment in exchange for course credits. The materials and procedure were the same as those in Experiment 1, except for the details of the presentation sequence and practice trials as well as the cues that were manipulated between subjects.

A trial proceeded as follows. A red dot location was presented first, followed sequentially by a blue dot location, then a green dot location, and, finally, a brown dot location. Each dot was presented for $1.5 \mathrm{sec}$ and was followed by a 1.0 -sec mask. The presentation of the four dot locations sequentially included two dots at a short radius and two dots at a long radius, with each dot presented in a different quadrant. Immediately following the presentation of the four dot locations, a variation of the Shepard-Metzler mental rotation task was presented in a forced choice format. Participants were presented with three 3-D target objects on-screen, the top one being the target and the bottom two being rotated versions of the same target or the mirror image of the target. Thus, the participant was asked to determine as rapidly as possible which of the rotated figures was the same as the target object by using the mouse. Feedback indicated whether the response was correct before the next trial of the Shepard-Metzler task began. Because this phase did not end until participants made a response, it could exceed the given 10 -sec minimum on a given trial. After the Shepard-Metzler task, the circular task field reappeared on-screen along with a color word (red, blue, green, or brown) printed in the corresponding color and presented at the top left of the screen, prompting which dot location to reproduce. Practice trials provided feedback and experience with both the four-dot presentation and the mental rotation task. After practice trials, there were a total of 64 experimental trials: 32 possible target locations for the red dots and 32 target locations distributed among the other three colors. We only analyzed the red dot locations, because they included the complete data set and had the greatest delay interval. (A pilot study had indicated that the first-presented dots were the most difficult to remember.) We presented the other 32 trials so that participants would not ignore the dot locations presented in the different colors. Because each location was estimated only once, inconsistency of estimation was not available in this experiment.

\section{Results}

Data analyses. Because of the difficulty of the task, an additional criterion was used to exclude participants whose overall performance on the task was poor. Only participants who demonstrated a correlation of .70 or greater between their responses and the actual dot locations were included. (In Experiments 1-3, no participant had a lower correlation than this criterion.) Out of the 42 participants in each cue condition, 5 participants were excluded in the 0 -cue condition, and 8 were excluded in the 4-cue condition. After excluding these participants, the same procedure used in Experiments 1-3 for eliminating outliers was applied. The mean percentage of outliers across conditions was $5.85 \%$ and ranged from $5.00 \%-6.61 \%$. For each dependent variable, a 2 (cue condition) $\times 2$ (radius) $\times 16$ (angle) mixedsubjects factorial ANOVA was conducted, and results are displayed in Table 5.

Angular bias. Figure 7 illustrates the angular bias results (and model fits) for the 0 - and 4-cue conditions. As 
Table 5

Degrees of Freedom $(d f)$ and $F$ Values for 2 (Cue Condition) $\times$ 2 (Radius) $\times 16$ (Actual Angle) Mixed Factorial ANOVAs of Experiment 4

\begin{tabular}{lcccc}
\hline \multicolumn{1}{c}{ Source } & \multicolumn{1}{c}{$d f$} & $\begin{array}{c}\text { Angular } \\
\text { Bias }\end{array}$ & $\begin{array}{c}\text { Radial } \\
\text { Bias }\end{array}$ & $\begin{array}{c}\text { Absolute } \\
\text { Error }\end{array}$ \\
\hline Cue $(\mathrm{C})$ & $(1,69)$ & 0.05 & 1.38 & 0.04 \\
Radius (R) & $(1,69)$ & $23.81^{* * *}$ & $531.97^{* * *}$ & 0.01 \\
$\mathrm{C} \times \mathrm{R}$ & $(1,69)$ & $4.14^{*}$ & 3.63 & $4.88^{*}$ \\
Angle (A) & $(15,1035)$ & $5.43^{* * *}$ & 1.53 & $21.31^{* * *}$ \\
$\mathrm{C} \times \mathrm{A}$ & $(15,1035)$ & $1.95^{*}$ & $2.00^{*}$ & 1.56 \\
$\mathrm{R} \times \mathrm{A}$ & $(15,1035)$ & 1.55 & $15.13^{* * *}$ & 1.26 \\
$\mathrm{C} \times \mathrm{R} \times \mathrm{A}$ & $(15,1035)$ & 0.92 & 1.01 & 0.92 \\
${ }^{*} p<.05 .{ }^{* *} p<.01 .{ }^{* * *} p<.001$. & &
\end{tabular}

was expected, the 0 -cue condition (panel A) showed the same default pattern found in Experiments 1-3. However, the pattern of bias is quite different for the 4-cue condition, as shown in panel B of Figure 7. First, the bias functions for the short and long radii differ. Second, the number and location of inferred prototypes appear to differ. These observations are supported by the analyses and modeling we report below.

It is instructive for us to compare the ANOVA results for Experiment 4 (Table 5) and Experiment 1 (Table 1), since the cue conditions were the same but the presentation conditions differed. Both experiments produced a significant main effect of angle, simply reflecting the presence of angular bias effects. However, in contrast with Experiment 1, a significant cue interaction was noted in Experiment 4, indicating that the pattern of angular bias was affected by the presence of cues. In addition, there was a significant effect of radius and a cue $\times$ radius interaction in Experiment 4, but not in Experiment 1. The radius effect indicated a higher negative bias for the short radius $(M=-6.10)$ than for the long radius $(M=-2.71)$. The cue $\times$ radius interaction reflected the radial effect occurring in the 4-cue condition but not in the 0 -cue condition.

Because interactions with cue were noted, the mean angular bias scores were fit to the fuzzy-boundary model separately for the 0 -cue and 4-cue conditions. Fitting procedures differed in two ways from those reported in Experiments 1-3. First, note that almost all angles produced a negative bias, reflecting a general tendency to displace targets in a clockwise direction. To model this effect, we included an additional parameter, $a$, which was used to redefine the intercept - that is, the value of greater bias when the target equals the prototype. Second, unlike Experiments 1-3, in which the fixedquadrants fuzzy-boundary model fit the data best, in Experiment 4, the flexible fuzzy-boundary model fit the data best. This was particularly needed in the 4-cue condition. Different versions of the flexible fuzzy-boundary model were generated by freeing different parameter values. Parameters were constrained as long as $R^{2}$ did not drop significantly.

For the 0 -cue condition, we found that the flexible fuzzyboundary model with seven parameters fit the data adequately $\left(R^{2}=.57\right)$. Table 6 presents the estimated parameter values in the corresponding row, and panel A of Figure 7 illustrates the model fit. As was expected, prototypes were modeled as being located very centrally within each quadrant. Note that the value of fine-grain memory weight was much lower than the values of fine-grain memory weight in the first three experiments, supporting the idea that finegrain memory is decreased when memory is severely disrupted. Reduced weighting of fine-grain memory is also reflected in the much greater angular bias in Experiment 4 than in the corresponding conditions of Experiment 1. As in Experiment 1, the value of the fuzzy-boundary parameter indicated fairly sharp boundaries. Thus, like the 0 -cue con-
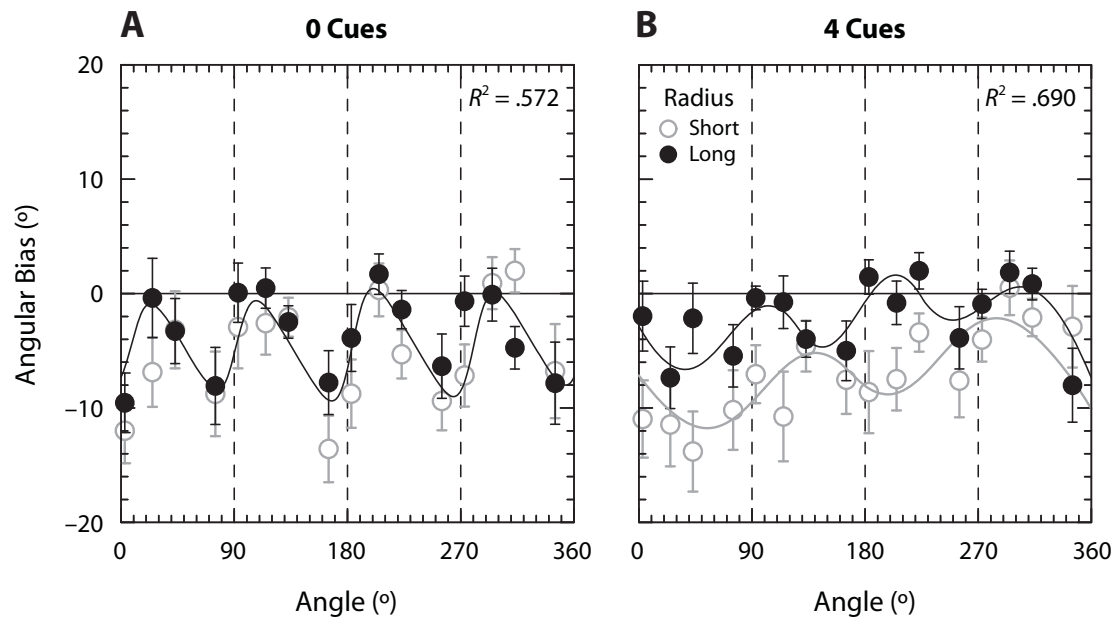

Figure 7. (A) The 7-parameter flexible fuzzy-boundary model on angular bias (one constant, four prototypes, one $\lambda$, and one $c$ ) for the 0 -cue condition. (B) The 10-parameter flexible fuzzy-boundary model on angular bias (for the long radius, one constant, one $\lambda$, three prototypes, two $c$ s varying with prototypes; for the short radius, one constant, one $\lambda$, two prototypes where one is the same as that for the short radius, two cs varying with prototypes that are the same as those for the long radius) for the 4-cue condition in Experiment 4. 
Table 6

Parameter Values and Fit Indices Modeling 0-Cue and 4-Cue Conditions Together for Angular Bias (Experiment 4)

\begin{tabular}{|c|c|c|c|c|c|c|c|c|c|c|}
\hline \multicolumn{2}{|c|}{ Condition } & \multicolumn{9}{|c|}{ Parameters } \\
\hline Cues & Radius & $p_{1}$ & $p_{2}$ & $p_{3}$ & $p_{4}$ & $\lambda$ & $c$ & $c_{3}$ & Constant & $R^{2}$ \\
\hline 0 & $\begin{array}{l}\text { Short } \\
\text { Long }\end{array}$ & $\begin{array}{c}49.08^{\circ} \\
"\end{array}$ & $\begin{array}{c}134.64^{\circ} \\
"\end{array}$ & $\begin{array}{c}233.34^{\circ} \\
"\end{array}$ & $\begin{array}{c}327.34^{\circ} \\
"\end{array}$ & $\begin{array}{c}.811 \\
"\end{array}$ & $\begin{array}{c}0.078 \\
"\end{array}$ & $\begin{array}{l}\mathrm{n} / \mathrm{a} \\
\mathrm{n} / \mathrm{a}\end{array}$ & $\begin{array}{c}-4.466 \\
"\end{array}$ & .572 \\
\hline 4 & $\begin{array}{l}\text { Short } \\
\text { Long }\end{array}$ & $\begin{array}{l}169.39^{\circ} \\
125.68^{\circ}\end{array}$ & $\begin{array}{c}\mathrm{n} / \mathrm{a} \\
226.87^{\circ}\end{array}$ & $\begin{array}{c}348.90^{\circ} \\
"\end{array}$ & $\begin{array}{l}\mathrm{n} / \mathrm{a} \\
\mathrm{n} / \mathrm{a}\end{array}$ & $\begin{array}{l}.798 \\
.631\end{array}$ & $\begin{array}{c}0.024 \\
"\end{array}$ & $\begin{array}{c}0.012 \\
"\end{array}$ & $\begin{array}{l}-7.053 \\
-2.197\end{array}$ & .690 \\
\hline
\end{tabular}

Note $-p_{1}, p_{2}, p_{3}, p_{4}$, prototype values; $\lambda$, weight of fine-grain memory; $c$, sensitivity parameter.

ditions of Experiments 1-3, the angular bias effects in the 0 -cue condition in Experiment 4 were adequately described by assuming the default category structure.

Consistent with the observed cue $\times$ radius interaction, we found that angular bias in the 4-cue condition had to be fit separately for each radius. The best flexible fuzzyboundary model included 10 free parameters fitting the data $\left(R^{2}=.69\right)$. Table 6 presents the estimated parameter values in the corresponding row, with panel B of Figure 7 illustrating the model fits. For the long radius, three prototypes were inferred from the data. The weight in the 4-cue condition was much lower than that in the 0 -cue condition, indicating that available cues did not necessarily help fine-grain memory for the long-radius dot locations. The sensitivity parameter, $c$, varied with prototypes and reflected fairly blunt boundaries. For the short-radius, the best fit included only two prototypes with corresponding sensitivity parameters from the long-radius condition (see Table 6). Even though the short radius indicated greater consistent clockwise bias across angle conditions than did the long radius, as indicated by the intercept for the short and long radius, the value of fine-grain memory weight was inferred to be higher for the short-radius dot locations than for the long radius. Overall, the model fits to the 4-cue conditions show a radically different categorical structure than that observed for the 0-cue condition or any of the conditions in Experiments 1-3. Specifically, cues appear to have induced fewer prototypes, with at least some of those prototypes being located along the horizontal axis - that is, nearer the cues.

Radial bias. Consistent with Experiments 1-3, there was again a strong effect for radius, with a radial bias toward the circumference for the short radius $(M=16.94)$ and a radial bias toward the center for the long radius $(M=-10.50)$. This pattern reflects a radial prototype located between short- and long-radius targets. Furthermore, a significant radius $X$ angle interaction indicated higher radial bias at the boundary of the first and fourth quadrant. However, because no specific predictions were made about the effects of angle on radial bias, we do not pursue this effect further. Finally, the only significant effect with cue was a significant cue $\times$ angle interaction. This interaction was small in magnitude, indicating that cue had little effect on radial bias when estimating locations under memory-taxing conditions.

Absolute error. Unlike in Experiment 1, a very large and significant main effect of angle was observed for absolute error (see Table 5). Although Experiments 2 and 3 produced significant effects of angle on absolute error as well, these were very small in comparison with that observed here. Thus, increasing memory constraints led to accuracy being strongly influenced by the angular location of the target. In addition, a significant cue $\times$ radius interaction was found: Short-radius targets led to less error in the 0 -cue condition $\left(M_{\text {short }}=33.26\right.$ vs. $\left.M_{\text {long }}=36.82\right)$, but this relationship was reversed in the 4-cue condition $\left(M_{\text {short }}=36.26\right.$ vs. $\left.M_{\text {long }}=33.05\right)$.

To better understand the large and significant effects of angle, we modeled the data using linear regression. The pattern of data suggested that error was minimized at the quadrant prototypes and along vertical and horizontal boundaries. To capture this effect, we used as predictor variables the logarithm of the angular distance from the targets to each of these locations (quadrant centroids at $45^{\circ}, 135^{\circ}, 225^{\circ}$, and $315^{\circ}$, and boundaries at $90^{\circ}, 180^{\circ}$, $270^{\circ}$, and $360^{\circ}$ ). We also included dummy variables that coded the radii for each condition. We used a backwardstepping procedure on the 64 data points, eliminating predictors that did not significantly reduce $R^{2}$ (at $\alpha=.05$ ) to determine a model that adequately fit the data.

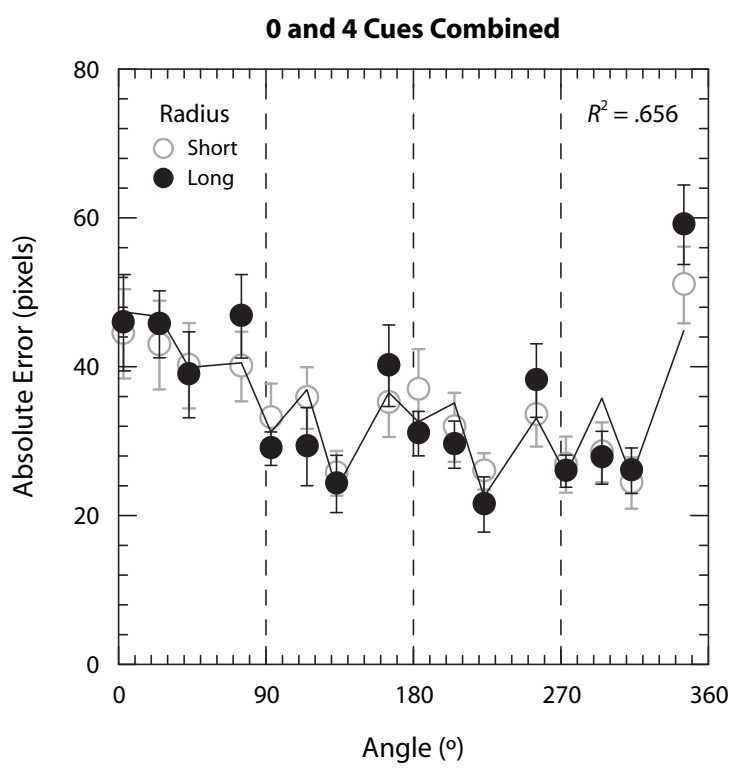

Figure 8. Mean absolute error scores with an 8-parameter regression model fit (one constant and all but one of the $\log$ (angle) predictors; i.e., the $360^{\circ}$ angle predictor was not included). The error bars indicate one standard error of the mean (Experiment 4). 
The final eight-parameter regression model included a constant and all but one of the log(angle) predictors (the $360^{\circ}$ angle predictor was not included). This model was significant $\left[F(7,56)=15.24, p<.001, R^{2}=.66\right.$ (adjusted $\left.\left.R^{2}=.61\right)\right]$. Figure 8 presents the absolute error data along with the function resulting from the regression model, combined for the cue conditions. The inferred constant was -113.38 , with all angle predictors receiving positive regression weights. These positive weights mean that absolute error was reduced when the distance to any of these angles was small, reflecting anchoring of fine-grain memory for proximal targets. This reduction is demonstrated in the prediction function by the sharp dips at each of the locations. This pattern of results suggests that in memory-demanding conditions, the horizontal and vertical boundaries imposed onto the circular task field, along with the centrally located prototypes, may be used as natural anchors to increase fine-grain memory.

\section{Discussion}

In contrast with results from Experiments 1-3, the available reference cues in Experiment 4 were used to create a categorization scheme that was different from the default scheme used when no cues were available. For the 0 -cue condition, model fits of angular bias were consistent with the default category scheme, as reported in previous experiments. For the 4-cue condition, model fits indicated that dot locations for the long and the short radii were estimated differently. Three prototypes were inferred for the longradius condition, located at $125^{\circ}, 225^{\circ}$, and $350^{\circ}$. Note that one of these locations corresponded to the right-horizontal cue, whereas the other two corresponded to central locations within the second and third quadrants. Two prototypes were inferred for the short-radius condition, located at $170^{\circ}$ and $350^{\circ}$. Note that these correspond roughly to the two horizontal cues. The model provided a good fit to the data, with no significant improvement if an additional prototype was included for either radius condition.

Although cues clearly altered the category structure, it is not clear why participants did not use all the available cues to restructure their categorical scheme. We suggest that cue usage to create category prototypes may be more effortful and demanding on working memory resources so that participants could not maintain four such cue-based prototypes. Indeed, the default category structure was clearly still viable, as demonstrated in the 0 -cue condition; thus, participants attempting to use cues may have focused on a subset, using only the horizontal cues. Finally, it is clear that the heavy memory demands of this task biased memory for location in a clockwise manner, reflected in the negative values of the intercepts ( $a$ s) in our modeling and in the fact that almost no targets were found to have a positive angular bias. This result was not anticipated and may reflect a time-linked error for this task.

The pattern of radial bias was consistent with the radial prototype locations being located between that of the long- and short-radius targets, again replicating findings from the first three experiments. Cues had little effect on radial bias. Of course, radial distortion might have varied with cues if the cues had varied in radial distances, which is something that future research could explore.

The results for absolute error reflected a very interesting pattern linked to angle. Regardless of cue condition, absolute error was reduced for targets located close to quadrant centers or boundaries. The pattern of effects of absolute error seems to indicate that in memory-demanding conditions, the horizontal and vertical boundaries imposed onto the circular task field as well as the centrally located prototypes may be used as natural anchors to increase fine-grain memory (see Spencer \& Hund, 2002, for additional evidence of the role of boundaries on error). Note that overall, the fine-grain memory is not improved with cue availability, which is inconsistent with the first three experiments. The fact that there was a cue $X$ angle interaction for angular bias but not for absolute error once again supports the utility of exploring both measures independently. Unlike Experiments 1-3, Experiment 4 did not demonstrate a main effect of cue on absolute error. This result suggests that in Experiment 4, the surrounding cues did not provide a way to hold fine-grain values in memory and hence produce more consistent and accurate estimates. Thus, the cue advantages on absolute error found in Experiments 1-3 do not appear to generalize to conditions in which memory may be more severely disrupted.

In summary, Experiment 4 demonstrated that under conditions of high memory disruption, cues appear to alter the categorization scheme away from the default quadrantbased scheme. However, we should point out that data nevertheless speak to the robustness of the default scheme used in a fixed environment. Indeed, quadrant-based prototypes were inferred for two of the three prototype locations for long-radius targets. This result suggests some flexibility in the categorization strategy of participants. Hund and Plumert's (2005) developmentally oriented investigation of stability and flexibility in categorization provides a framework for further exploration of this issue.

\section{GENERAL DISCUSSION}

Our primary goal in this set of studies was to better understand how cues that are external to the task field may influence memory for location when orientation to the task field is fixed. A fixed orientation to an environment occurs when neither the individual nor the stimulus within the task field change orientation, such as when a person is monitoring a screen in an experiment, or when an air traffic controller is monitoring a radar screen. In these situations, a cue-independent category scheme is sufficient to encode the stimulus location, so cue information may be seen as extraneous or surplus information. This is not the case when the orientation of the participant to the environment changes dynamically, often making cue-based encoding essential to remembering location, as in the Morris water maze task. Our prior research demonstrated that when the task field was rotated on a majority of trials, cues played a critical role in encoding locations into memory, even on the minority of trials in which there was no rotation (Fitting et al., 2007). That same research demonstrated no effects 
of including either 1 or 3 cues on memory for location when a fixed orientation was present on all trials.

The present studies provided a more rigorous test of the role of cues in remembering locations in a static environment by manipulating more extensively the number of cues, the location of cues, and also the memory demands of the task. We were particularly interested in two roles cues might play. One role is to organize the categorical structure used to remember location, which should be reflected in the angular bias measure. A second role is to serve as a way to bolster fine-grain memory, which should be primarily reflected in effects in absolute error and inconsistency of estimation measures (and to a lesser degree in angular bias). We consider these two roles below.

\section{The Role of Cues in Structuring Memory}

As shown in the theoretical functions of Figure 1, using cues as category prototypes (panels B and C) can lead to much different patterns of angular bias than when quadrant centroids are used as category prototypes (panel A). The quadrant-based pattern of bias has been found time and again in a myriad of studies examining spatial memory within a small geometric task field (Engebretson \& Huttenlocher, 1996; Huttenlocher et al., 1991; Spencer \& Hund, 2002; Wedell et al., 2007). It is then striking that Fitting et al. (2007) were able to generate the cue-based patterns shown in panels $\mathrm{B}$ and $\mathrm{C}$ of Figure 1 by rotating the orientation of the task field on a majority of trials. We believe that this result parallels the distinction between response memory and place memory found in studies of the Morris water maze. In both cases, when cues become essential for orienting to the task field, they then serve the role of organizing memory for location. In the dot location task, the angular bias measure is diagnostic of the categorical structure being used to encode location. Thus, modeling angular bias gives us an insight into the potential use of cues to organize memory.

Under what circumstances might cues be used to structure memory for location within a static task environment? Although Fitting et al. (2007) had demonstrated that including 1 or 3 external cues does not lead to a cuebased organization of memory in the static environment, it was unclear whether this result would generalize to the situation when there were more cues available. Following a rational view of memory encoding, Huttenlocher et al. (1991) pointed out that the bias induced by using categorical memory may prove adaptive in that it reduces overall error in much the same way regressing toward the mean reduces error in prediction more generally. All else being equal, then, adopting a cue-based categorical structure in the previously reported study would have led to more error, since the cue structure would generate fewer categories and, hence, greater bias. In Experiments 1-3, we systematically increased the number of available cues to test whether this manipulation would lead to the use of cues in organizing memory. The locations of the cues were designed to provide a pattern of bias easily distinguished from the default encoding scheme typically observed. In each case, we found no evidence that the cues were being used to structure memory. The same four-quadrant model of angular bias was found in each condition of Experiments $1-3$. Thus, this default categorical structure appears to be quite robust.

The failure to use cue-based categories in Experiments $1-3$ presents a challenge to the rational basis of memory. All else being equal, error tends to be reduced when more categories are used, so it seems maladaptive not to use these cue-based categories. However, this conclusion presupposes that participants are capable of effectively using all given cues to establish a category structure. If the default four-category scheme arises out of automatically processing stimuli within a viewer-based or geometric-based frame of reference, then switching to a cue-based structure may require additional resources that may limit the number of categories that can be effectively used.

The results of Experiment 4 provide some support for this supposition. In Experiment 4, memory for location was severely taxed by presenting multiple targets and including a filled delay interval designed to occupy spatial working memory. Remarkably, participants in the 0-cue condition produced a pattern of bias that was not particularly different from that generated by participants in the much less taxing conditions of Experiments 1-3. The default coding scheme appeared to work quite well. On the other hand, those participants who were presented with the 4 cues at the axes of the circle appeared to have less success in structuring memory for location. Although some cue locations were incorporated into the category structure, default quadrant locations were also used. Furthermore, the number of prototypes that were inferred in this condition was just two for the short-radius targets (which are typically more poorly remembered) and three for the long-radius targets. One possible explanation of this pattern of results is that the incorporation of cues into the category structure is a more effortful process that participants were not able to fully implement. If this is the case, then failure to use multiple cues to structure memory may not be a failure of rationally motivated memory, but it may simply reflect a limit on cue-based processing. Further support for this idea derives from an analysis by Fitting et al. (in press) of prototypes for rotation trials. On these more memory-taxing trials, they found that participants appeared to only use 2 cues when 3 were available. Thus, as memory demands increase, it may become more difficult to incorporate multiple cuebased categories in memory.

In conclusion, it appears that the four-quadrant categorical encoding of spatial location is quite robust when orientation to the environment is fixed. This conclusion is reinforced by the fact that it required severe taxing of memory to produce some evidence of incomplete cue usage in Experiment 4. The default encoding structure may derive from either a viewer-based perspective that encodes up-down and right-left distinctions, or it may derive from a more geometric-based orientation. Our results cannot distinguish between these two possibilities. However, a recent study by Wedell et al. (2007) provides some 
support for the viewer-based frame of reference determining the default structure. In that experiment, the shape of the task field was manipulated by presenting dot locations within a square, triangle, or pentagon. A geometric-based coding system would seem to entail a change in the coding scheme with such a strong change in the geometric shape. However, a four-category scheme was noted, consistent with coding left-right and up-down viewer-based axes of orientation. Future research is needed to further distinguish the usage of these two types of cue-independent frames of reference (for more, see Cheng \& Newcombe, 2005; McNamara, Rump, \& Werner, 2003).

\section{The Role of Cues in Anchoring Memory}

Despite a lack of cue effects on the categorization scheme, the present studies provided strong evidence that even in a static environment, cues have an impact on memory for location. In Experiments 1-3, absolute error and inconsistency of estimation were reduced in the cue conditions. In Experiments 2 and 3, these effects were demonstrated to be stronger for the long-radius targets more proximal to the cues. We interpret these results by assuming that cues serve as anchors that enhance the fine-grain memory representation, especially for proximal locations. In addition, Experiments 2 and 3 revealed cue effects on the slopes of the angular bias functions that can be interpreted in terms of greater weighting of fine-grain memory with available cues. Experiment 3 also demonstrated that cues may serve as buffers against the effects of delay for proximal target, as seen in the reduced slopes for the longdelay conditions in Figure 4.

The use of cues as anchors to improve fine-grain memory in the first three experiments supports the view of the rational nature of memory processes in spatial cognition (Huttenlocher et al., 1991). So why did cues, then, not reduce absolute error in Experiment 4? One possibility is that the effortful integration of cues in restructuring category memory may have resulted in greater bias, which may have resulted in greater absolute error. This interpretation is speculative and requires further testing. Experiment 4, however, also demonstrated a striking pattern of reduced error at prototype and boundary locations that was not present in Experiments 1-3. This pattern resembles the kind of highly localized absolute error reduction that we find in rotation conditions when memory is taxed (Fitting et al., in press). The reduced error at boundaries is consistent with boundary effects reported by Spencer and colleagues (Simmering et al., 2006; Spencer \& Hund, 2002; Spencer et al., 2006). The combined findings suggest that when memory is taxed, anchoring of fine-grain memory may be much more localized.

In conclusion, our research demonstrates that it is important to study experimental manipulations across a wide range of memory and environmental conditions to better understand the nature of how these manipulations affect spatial memory. Cues may restructure category memory when orientation to the task field is uncertain, but they rarely serve that function in a fixed environment. In both static and dynamic environments, cues may also serve to anchor fine-grain memory and thus improve overall accuracy. Our research to date has created a dynamic envi- ronment by rotating the task field itself. Future research should investigate whether the reported effects can be replicated in other dynamic tasks, such as when one navigates to a location or when one is asked to adopt a different perspective within an environment.

\section{AUTHOR NOTE}

Address correspondence to S. Fitting, Department of Pharmacology \& Toxicology, Virginia Commonwealth University, School of Medicine, Room 437, Kontos Medical Sciences Building, 1217 East Marshall Street, Richmond, VA 23298 (e-mail: sfitting@vcu.edu).

\section{REFERENCES}

Allen, G. L., \& Haun, D. B. M. (2004). Proximity and precision in spatial memory. In G. Allen (Ed.), Human spatial memory: Remembering where (pp. 41-61). Mahwah, NJ: Erlbaum.

Cheng, K., \& Newcombe, N. S. (2005). Is there a geometric module for spatial orientation? Squaring theory and evidence. Psychonomic Bulletin \& Review, 12, 1-23.

EASTON, R. D., \& SHOLL, M. J. (1995). Object-array structure, frames of reference, and retrieval of spatial knowledge. Journal of Experimental Psychology: Learning, Memory, \& Cognition, 21, 483-500.

Engebretson, P. H., \& Huttenlocher, J. (1996). Bias in spatial location due to categorization: Comment on Tversky and Schiano. Journal of Experimental Psychology: General, 125, 96-108.

FITTING, S. (2005). Memory for spatial location: Cue effects as a function of field rotation. Unpublished master's thesis, University of South Carolina, Columbia.

Fitting, S., Wedell, D. H., \& Allen, G. L. (2005). Memory for spatial location: Influences of environmental cues and task field rotation. In A. G. Cohn \& D. M. Mark (Eds.), COSIT 2005, LNCS 3693 (pp. 459474). Berlin: Springer.

Fitting, S., Wedell, D. H., \& Allen, G. L. (2007). Memory for spatial location: Cue effects as a function of field rotation. Memory \& Cognition, 35, 1641-1658.

Fitting, S., Wedell, D. H., \& Allen, G. L. (in press). External cue effects on memory for spatial location within a rotated task field. Spatial Cognition \& Computation.

Greenhouse, S. W., \& GeIsser, S. (1959). On methods in the analysis of profile data. Psychometrika, 32, 95-112.

Haun, D. B. M., Allen, G. L., \& Wedell, D. H. (2005). Bias in spatial memory: A categorical endorsement. Acta Psychologica, 118, 149-170.

Hund, A. M., \& Plumert, J. M. (2002). Delay-induced bias in children's memory for location. Child Development, 73, 829-840.

Hund, A. M., \& Plumert, J. M. (2005). The stability and flexibility of spatial categories. Cognitive Psychology, 50, 1-44.

Huttenlocher, J., Hedges, L. V., \& Duncan, S. (1991). Categories and particulars: Prototype effects in estimating spatial location. Psychological Review, 98, 352-376.

McNamara, T. P., Rump, B., \& Werner, S. (2003). Egocentric and geocentric frames of reference in memory of large-scale space. Psychonomic Bulletin \& Review, 10, 589-595.

Merchant, H., Fortes, A. F., \& Georgopoulos, A. P. (2004). Shortterm memory effects on the representation of two-dimensional space in the rhesus monkey. Animal Cognition, 7, 133-143.

Morris, R. G. M. (1981). Spatial localization does not require the presence of local cues. Learning \& Motivation, 12, 239-260.

Mou, W. M., \& McNamara, T. P. (2002). Intrinsic frames of reference in spatial memory. Journal of Experimental Psychology: Learning, Memory, \& Cognition, 28, 162-170.

ShePARD, R. N. (1987). Toward a universal law of generalization for psychological science. Science, 237, 1317-1323.

Simmering, V. R., SPencer, J. P., \& Schöner, G. (2006). Referencerelated inhibition produces enhanced position discrimination and fast repulsion near axes of symmetry. Perception \& Psychophysics, 68, 1027-1046.

SPENCER, J. P., \& Hund, A. M. (2002). Prototypes and particulars: Geometric and experience-dependent spatial categories. Journal of Experimental Psychology: General, 131, 16-37. 
Spencer, J. P., \& Hund, A. M. (2003). Developmental continuity in the processes that underlie spatial recall. Cognitive Psychology, 47, 432-480.

Spencer, J. P., Simmering, V. R., \& Schutte, A. R. (2006). Toward a formal theory of flexible spatial behavior: Geometric category biases generalize across pointing and verbal response types. Journal of Experimental Psychology: Human Perception \& Performance, 32, 473-490.
WANG, R. F., \& Spelke, E. S. (2000). Updating egocentric representations in human navigation. Cognition, 77, 215-250.

Wedell, D. H., Fitting, S., \& Allen, G. L. (2007). Shape effects on memory for location. Psychonomic Bulletin \& Review, 14, 681-686.

WERnER, S., \& DiEDrichSEN, J. (2002). The time course of spatial memory distortions. Memory \& Cognition, 30, 718-730.

WILKINSON, L. (1989). SYSTAT: The system for statistics. Evanston, IL: SYSTAT, Inc.

\section{APPENDIX}

Modeling of the data was based on an elaboration of the Huttenlocher et al. (1991) category-adjustment model that we have described elsewhere (Fitting et al., 2005, 2007). This model assumes that targets near a boundary may recruit more than one prototype (Haun et al., 2005); hence, we describe the model as having fuzzy boundaries.

The basic fuzzy-boundary model modifies the category-adjustment model (Equation 1) in the following way:

$$
E[\text { Bias }]=\lambda \mu+(1-\lambda) \Sigma \operatorname{Pr}\left(p_{j} \mid \mu\right) p_{j}-\mu .
$$

The key difference between Equation A1 and the basic Huttenlocher et al. (1991) model (Equation 1 in the main text) is that the prototype weighting $(1-\lambda)$ is applied to each prototype in Equation A1, as modified by the probability of prototype retrieval given the stimulus. The two models that we describe below differ in the prototype recruitment function that describes the probability of prototype retrieval.

We developed two versions of the fuzzy-boundary model. The fixed-quadrants fuzzy-boundary model assumes the default four-quadrant category structure, with prototype recruitment based on the relative similarity of the stimulus angle to midpoints of the four categories. We incorporate the exponential decay function as our similarity function, since it has been successfully applied elsewhere (Shepard, 1987). Within this framework, the probability of prototype recruitment can be described as follows:

$$
\operatorname{Pr}\left(p_{j} \mid \mu\right)=\frac{\exp \left[-c\left|\mu-.5\left(t_{\min , j}+t_{\max , j}\right)\right|\right]}{\sum \exp \left[-c\left|\mu-.5\left(t_{\min , k}+t_{\max , k}\right)\right|\right]},
$$

where similarity is calculated relative to the midpoint of the category, using the average of the lower boundary $\left(t_{\min }\right)$ and upper boundary $\left(t_{\max }\right)$. The fixed-quadrants fuzzy-boundary model incorporates Equation A2 into Equation 1, modifying prototype weighting by the probability of prototype recruitment, $\operatorname{Pr}\left(p_{j} \mid \mu\right)$. Note that an important constraint of this model is that the prototype for a quadrant must reside within that quadrant. Further details for fitting this model are found in Fitting et al. (2005, 2007).

The alternative version of our model is one in which the number and locations of the prototypes are free to vary. Because this model was developed with the idea that cues would serve as category prototypes, we have called it the flexible fuzzy-boundary model. In this model, boundaries are inferred by assuming they fall at equal distances from category prototypes. Accordingly, the prototype recruitment equation is based on the similarity of the stimulus to the prototype as follows:

$$
\operatorname{Pr}\left(p_{j} \mid \mu\right)=\frac{\exp \left(-c\left|\mu-p_{j}\right|\right)}{\sum \exp \left(-c\left|\mu-p_{k}\right|\right)} .
$$

The flexible fuzzy-boundary model incorporates Equation A3 into Equation 1, modifying prototype weighting by the probability of prototype recruitment, $\operatorname{Pr}\left(p_{j} \mid \mu\right)$. Again, the specific details on fitting this model are found in Fitting et al. (2005, 2007).

In summary, the fuzzy-boundary models essentially add just one parameter to the basic-category adjustment model, $c$, which determines the sharpness of the boundary. In this article, adequate fits by the fixed-quadrants fuzzy-boundary model to angular bias in the 4-, 8-, and 24-cue conditions would indicate the use of a cueindependent categorization scheme. On the other hand, if cues were having a strong influence on the categorization scheme in these experiments, then one would expect the flexible fuzzy-boundary model to provide a better fit and reflect different numbers of categories or a different orientation of those categories. 